\title{
Equilibrium pricing bounds on option prices
}

\author{
Marie Chazal $^{a}$ and Elyès Jouini ${ }^{b}$ \\ ${ }^{a}$ CREST, France 83 Department of Mathematics, ETH Zentrum, CH-8092 Zurich \\ mchazal@math.ethz.ch \\ ${ }^{b}$ CEREMADE, Université Paris-Dauphine, Place du Maréchal de Lattre de Tassigny, \\ 75775 Paris Cedex 16, France 83 Institut Universitaire de France \\ jouini@ceremade.dauphine.fr
}

\begin{abstract}
We consider the problem of valuing European options in a complete market but with incomplete data. Typically, when the underlying asset dynamics is not specified, the martingale probability measure is unknown. Given a consensus on the actual distribution of the underlying price at maturity, we derive an upper bound on the call option price by putting two kind of restrictions on the pricing probability measure. First, we put a restriction on the second risk-neutral moment of the underlying asset terminal value. Second, from equilibrium pricing arguments one can put a monotonicity restriction on the Radon-Nikodym density of the pricing probability with respect to the true probability measure. This density is restricted to be a nonincreasing function of the underlying price at maturity. The bound appears then as the solution of a constrained optimization problem and we adopt a duality approach to solve it. We obtain a weak sufficient condition for strong duality and existence for the dual problem to hold, for options defined by general payoff functions. Explicit bounds are provided for the call option. Finally, we provide a numerical example.
\end{abstract}

Key words: Option bounds, equilibrium prices, conic duality, semi-infinite programming OR Subjects: Finance: Asset pricing. Programming: Infinite dimensional. Utility/preference: Applications

Area of Review: Financial engineering 


\section{Introduction}

A central question in finance consists of finding the price of an option, given information on the underlying asset. We investigate this problem in the case where the information is imperfect. More precisely, we are interested in determining the price of an option without making any distributional assumption on the price process of the underlying asset. It is well known that, in a complete financial market, by the no-arbitrage condition, the price of an option is given by the expectation of its discounted payoff under the risk-neutral probability, i.e. the unique probability measure that is equivalent to the historical one, and under which the discounted price processes of the primitive assets are martingales. The identification of this pricing probability requires the perfect knowledge of the primitive assets dynamics. Hence, in our restricted information context, one cannot use the exact pricing rule. But, one can always search for a bounding principle for the price of an option. One question is how to compensate part of the lack of information on the underlying asset dynamics? Assuming (lowly) knowledge on investors' preferences, i.e. risk aversion, and using equilibrium arguments, one obtains a qualitative information of the risk-neutral probability density, on which our bounding rule is based. It has a great advantage from an empirical point of view since it requires no market data. Our rule also uses a quantitative information on the underlying asset but only on its price at maturity, as it is done in the pioneer works of Lo (1987).

Lo initiated a literature on semi-parametric bounds on option prices. He derived upper bounds on the prices of call and put options depending only on the mean and variance of the stock terminal value under the risk-neutral probability : he obtained a closed-form formula for the bound as a function of these mean and variance. This work has been extended to the case of conditions on the first and the $n$th moments, for a given $n$, by Grundy (1991). Bertsimas and Popescu (2002) generalized these results to the case of $n \geq 2$ moments restrictions. When the payoff is a piecewise polynomial, the bounding problem can be rewritten, by considering a dual problem, as a semi-definite programming problem and thus can be solved from both theoretical and numerical points of view. 
Gotoh and Konno (2002) proposed an efficient cutting plane algorithm which solves the semi-definite programming problem associated to the bound depending on the first $n$ moments. According to their numerical results, the upper bound of Lo is significantly tightened by imposing more than 4 moments conditions. Since the mean of the terminal stock discounted price under the martingale measure is given by the current stock price, the first moment condition is totally justified. However, the knowledge of the $n \geq 2$ moments under the risk-neutral probability is a little illusive. We restrict ourselves to put constraints on the two first risk-neutral moments and use some qualitative information on the risk-neutral measure in order to improve the bound of Lo. In Black-Scholes like models the variance of the stock price is the same under the true and the risk-neutral probabilities. This provides then a justification for the knowledge of the second moment under the risk-neutral probability.

The restriction that we put on the martingale measure comes from equilibrium and hence preferences considerations : in an arbitrage-free and complete market with finite horizon $T$, the equilibrium can be supported by a representative agent, endowed with one unit of the market portfolio, that maximises the expected utility $U$ of his terminal wealth $X_{T}$ under his budget constraint. The first order condition implies that the RadonNikodym density with respect to the true probability measure of the martingale measure, $\frac{d \mathbb{Q}}{d \mathbb{P}}$, is positively proportional to $U^{\prime}\left(X_{T}\right)$. Under the usual assumption that agents are risk-averse, the utility function $U$ is concave. It is therefore necessary that the density $\frac{d \mathbb{Q}}{d \mathbb{P}}$ is a nonincreasing function of the terminal total wealth $X_{T}$. When the derivative asset under consideration is written on the total wealth or on some index seen as a proxy of the total wealth, one can restrict his attention to a pricing probability measure that has a nonincreasing Radon-Nikodym density with respect to the actual probability measure (remark that in the Black-Scholes model, the risk-neutral density satisfies this monotonicity condition if and only if the underlying drift is upper than the risk-free rate, which is a necessary and sufficient condition for the stock to be positively held). This ordering principle on the martingale probability measure with respect to the underlying asset price has been introduced by Perrakis and Ryan (1984). Together with Ritchken 
(1985), they launched an important part of the literature on bounding option prices by taking into account preferences properties as for instance risk-aversion. Bizid, Jouini and Koehl (1999) and Jouini and Napp (1998) obtained, in different settings, that this ordering principle is a necessary condition for options prices to be compatible with an equilibrium. Following their terminology, we call "equilibrium pricing upper bound" on the price of an option maturing at the terminal date, a bound that is obtained under the restriction that the Radon-Nikodym density of the pricing probability measure is in reverse order with the underlying terminal value (see also Jouini 2003 for the definitions of equilibrium prices, equilibrium pricing intervals in incomplete markets and their convergence properties). As an example, $B_{P \& R}:=\sup \left\{E^{\mathbb{Q}}\left[\psi\left(S_{T}\right)\right], \mathbb{Q}: E^{\mathbb{Q}}\left[S_{T}\right]=S_{0}, d \mathbb{Q} / d \mathbb{P} \searrow\right.$ w.r.t. $\left.S_{T}\right\}$ is an equilibrium pricing upper bound on the price of an option with payoff $\psi\left(S_{T}\right)$, when we only know the distribution of the terminal stock price $S_{T}$, under the true probability measure $\mathbb{P}$. We obtain that, for the call option, $B_{P \& R}=\frac{S_{0}}{E^{\mathbb{P}}\left[S_{T}\right]} E^{\mathbb{P}}\left[\psi\left(S_{T}\right)\right]$. This expression has already been obtained as a bound on the price of a call option, starting from different considerations, by Levy (1985), Perrakis and Ryan (1984) and Ritchken (1985). Levy (1985) obtained it as the minimum price for the call above which there exists a portfolio, made up of the stock and the riskless asset, of which the terminal value dominates, in the sense of second order stochastic dominance, the terminal value of some portfolio with the same initial wealth but made of call units. Perrakis and Ryan (1984) derived it as the upper bound on a call option arbitrage price, for stock price distributions such that the normalized conditional expected utility for consumption is nonincreasing in the stock price. Ritchken (1985) derived the same upper bound, with a finite number of states of the world, by restricting the state-contingent discount factors to be in reverse order with the aggregate wealth which is itself assumed to be nondecreasing with the underlying security price. When interpreting the state $j$ discount factor as the discounted marginal utility of wealth of the representative agent in state $j$, this restriction corresponds to the concavity of the representative utility function. The concavity assumption accounts for risk-aversion and means that agent have preferences that respect the second order stochastic dominance principle. By extension, in an expected-utility model, preferences 
are said to respect the $n$th order stochastic dominance rule if the utility function is such that its derivatives are successively nonnegative and nonpositive up to $n$th order. Ritchken and Kuo (1989), Basso and Pianca (2001) proposed the application of such rules to put additional restrictions of the state discount factors and thus improve Ritchken's bounds. These works are also to be related to more recent results, in a continuous state of the world framework, by e.g. Constantinides and Perrakis (2002) who derived stochastic dominance upper (lower) bounds on the reservation write (purchase) price of call and put options in a multi-period economy and in the presence of transaction costs.

Our main contribution is to provide an equilibrium pricing upper bound for the price of a European call option, given a consensus on the actual distribution of the underlying terminal value and given its second risk-neutral moment. The novelty is in combining moment constraints and the monotonicity condition on the Radon-Nikodym density of the risk-neutral probability with respect to the true probability.

We adopt a conic duality approach to solve the constrained optimization problem corresponding to our bounding problem. By the use of some classical result in moments theory, given in Shapiro (2001), we obtain some sufficient condition for strong duality and existence in the dual problem to hold, for derivative assets defined by general payoff functions. Explicit bounds are derived for the call option, by solving the dual problem which is a linear programming problem with an infinite number of constraints. This also allows us to solve the primal problem. We observe on some numerical example that Lo's bound is at least as tightened by the qualitative restriction on the risk-neutral probability measure as by the quantitative information on the third and fourth risk-neutral moments of the underlying asset.

The paper is organized as follows. Section 1 is devoted to the equilibrium pricing upper bound formulation. The duality results are provided in Section 2 and the equilibrium pricing upper bound for the call option is derived in Section 3. We provide a numerical example in Section 4 and finally make concluding remarks. All proofs are given in a mathematical appendix. 


\section{The model formulation}

We consider a financial market with a finite horizon $T$, with assets with prices defined on a given probability space $(\Omega, \mathcal{F}, \mathbb{P})$. One of these asset is riskfree. We assume, without loss of generality and for sake of simplicity, that the riskfree rate is 0 .

The market is assumed to be arbitrage-free, complete and at the equilibrium. Hence there exists a probability measure $\overline{\mathbb{Q}}$, equivalent to $\mathbb{P}$, under which the assets prices processes are martingales. Since the market is at equilibrium, the Radon-Nikodym density $\frac{d \overline{\mathbb{Q}}}{d \mathbb{P}}$ is a nonincreasing function of the terminal total wealth or equivalently of the terminal value of the market portfolio. We want to put an upper bound on the price of an option written on the market portfolio or on on some index, which can be seen as a proxy of the market portfolio.

We denote by $m$ the price of the underlying asset at time 0 and by $S_{T}$ its price at the terminal time. We assume that $m \in \mathbb{R}^{+}$. The price $S_{T}$ is assumed to be a nonnegative random value on $(\Omega, \mathcal{F}, \mathbb{P})$ which is square integrable under $\mathbb{P}$ and $\overline{\mathbb{Q}}$. We suppose that its distribution under $\mathbb{P}$ has a density with respect to the Lebesgue measure, which is known. This density is denoted by $f$ and it is assumed to be positive on $[0, \infty)$. We denote by

$$
p_{1} \triangleq \int_{0}^{\infty} x f(x) d x \text { and } p_{2} \triangleq \int_{0}^{\infty} x^{2} f(x) d x
$$

the first and second moments of $S_{T}$ under $\mathbb{P}$.

We have $m=E^{\overline{\mathbb{Q}}}\left[S_{T}\right]$ and we set $\delta:=E^{\overline{\mathbb{Q}}}\left[S_{T}^{2}\right]$.

We further assume that $S_{T}$ is an increasing function of the terminal value of the market portfolio. Hence, there exists a function $\bar{g}$ which is positive and nonincreasing on $(0, \infty)$ such that $\frac{d \overline{\mathbb{Q}}}{d \mathbb{P}}=\bar{g}\left(S_{T}\right)$ and such that the functions $f \bar{g}, x f \bar{g}$ and $x^{2} f \bar{g}$ are in $L^{1}(0, \infty)$ and satisfy

$$
\int_{0}^{\infty} f(x) \bar{g}(x) d x=1, \int_{0}^{\infty} x f(x) \bar{g}(x) d x=m \text { and } \int_{0}^{\infty} x^{2} f(x) \bar{g}(x) d x=\delta .
$$

Given a payoff function $\psi$ such that the functions $\psi f$ and $\psi f \bar{g}$ are in $L^{1}(0, \infty)$, 
we denote by $\mathcal{X}$ the vector space generated by the nonnegative measures $\mu$ on $([0, \infty)$, $\mathcal{B}([0, \infty)))$, such that the functions $\psi f, f, x f$ and $x^{2} f$ are $\mu$-integrable. We assume that 0 is a Lebesgue point of both $\psi f$ and $f$, i.e.

$$
\lim _{r \rightarrow 0} \frac{1}{r} \int_{(0, r)}|\psi(x) f(x)-\psi(0) f(0)| d x=\lim _{r \rightarrow 0} \frac{1}{r} \int_{(0, r)}|f(x)-f(0)| d x=0 .
$$

The space $\mathcal{X}$ therefore contains the Dirac measure at $0, \delta_{0}$. Let $\mathcal{C}$ be the convex cone of $\mathcal{X}$ generated by $\delta_{0}$ and by the elements $\mu$ of $\mathcal{X}$ that have nonnegative and nonincreasing densities on $(0, \infty)$.

We put the following upper bound on the equilibrium price of an option with payoff $\psi\left(S_{T}\right)$

$$
(P) \quad \sup _{\mu \in \mathcal{C}_{m, \delta}} \int_{0}^{\infty} \psi(x) f(x) d \mu(x)
$$

where $\mathcal{C}_{m, \delta}$ is the set of $\mu \in \mathcal{C}$ which satisfy

$$
\int_{0}^{\infty} f(x) d \mu(x)=1, \int_{0}^{\infty} x f(x) d \mu(x)=m \text { and } \int_{0}^{\infty} x^{2} f(x) d \mu(x)=\delta .
$$

We denote by $\operatorname{val}(P)$ the value of problem $(P)$.

Remark 1.1 Let $\mathcal{G}$ be the set of nonnegative, nonincreasing functions $g$ on $(0, \infty)$ such that $\psi f g, f g, x f g$ and $x^{2} f g$ are in $L^{1}(0, \infty)$. Any element $\mu$ of $\mathcal{C}$ can be decomposed as follows: $d \mu=\alpha d \delta_{0}+g d x$ where $\alpha \in \mathbb{R}^{+}$and $g \in \mathcal{G}$.

Remark 1.2 One can always assume that $\psi(0)=0$. Indeed, if $(\tilde{P})$ is the problem associated to $\psi-\psi(0)$ then, it is clear that $\operatorname{val}(P)=\operatorname{val}(\tilde{P})+\psi(0)$. Therefore, in the sequel, we work under the assumption that

$$
\psi(0)=0
$$




\section{The dual problem formulation}

In this section, we formulate the dual problem of $(P)$. Let $\mathcal{X}^{\prime}$ be the vector space generated by $\psi f, f, x f$ and $x^{2} f$. The spaces $\mathcal{X}$ and $\mathcal{X}^{\prime}$ are paired by the following bilinear form

$$
(h, \mu) \in \mathcal{X}^{\prime} \times \mathcal{X} \longmapsto \int_{0}^{\infty} h(x) d \mu(x)
$$

Let us introduce the polar cone of $\mathcal{C}$ :

$$
\mathcal{C}^{*}=\left\{h \in \mathcal{X}^{\prime} \mid \int_{0}^{\infty} h(x) d \mu(x) \geq 0, \forall \mu \in \mathcal{C}\right\}
$$

In all the sequel, when considering $v \in \mathbb{R}^{3}$, we will denote $v \triangleq\left(v_{0}, v_{1}, v_{2}\right)$.

It is clear that for all $\lambda \in \mathbb{R}^{3}$ such that $\lambda_{0} f+\lambda_{1} x f+\lambda_{2} x^{2} f-\psi f \in \mathcal{C}^{*}$, and for all measure $\mu \in \mathcal{C}_{m, \delta}$ we have

$$
\int_{0}^{\infty} \psi(x) f(x) d \mu(x) \leq \lambda_{0}+\lambda_{1} m+\lambda_{2} \delta
$$

It is therefore natural to consider the following problem

$$
\begin{aligned}
& \inf _{\lambda \in \mathbb{R}^{3}} \lambda_{0}+\lambda_{1} m+\lambda_{2} \delta \\
& \text { subject to } \lambda_{0} f+\lambda_{1} x f+\lambda_{2} x^{2} f-\psi f \in \mathcal{C}^{*}
\end{aligned}
$$

We denote by $\operatorname{val}(D)$ the value of problem $(D)$ and by $\operatorname{Sol}(D)$ the set of solutions to $(D)$, i.e.

$$
\operatorname{Sol}(D) \triangleq\left\{\lambda \in \mathbb{R}^{3} \mid \lambda_{0} f+\lambda_{1} x f+\lambda_{2} x^{2} f-\psi f \in \mathcal{C}^{*} \text { and } \lambda_{0}+\lambda_{1} m+\lambda_{2} \delta=\operatorname{val}(D)\right\}
$$

From Proposition 3.4 in Shapiro (2001), we have some strong duality between the two problems under the condition given in the following proposition. 
Let

$$
F \triangleq\left\{v \in \mathbb{R}^{3} \mid \exists \mu \in \mathcal{C}: v=\left(\int_{0}^{\infty} f(x) d \mu(x), \int_{0}^{\infty} x f(x) d \mu(x), \int_{0}^{\infty} x^{2} f(x) d \mu(x)\right)\right\} .
$$

Proposition 2.1 If $(1, m, \delta) \in \operatorname{Int}(F)$ then $\operatorname{val}(P)=\operatorname{val}(D)$. If this common value is further finite, then the set of solutions to $(D)$ is non-empty and bounded. Conversely, if $\operatorname{val}(D)$ is finite and the set of solutions to $(D)$ is non-empty and bounded then $(1, m, \delta) \in$ $\operatorname{Int}(F)$.

In Proposition 2.2 below, we determine $F$, we check that $(1, m, \delta)$ is in $F$ and we provide some sufficient condition for $(1, m, \delta)$ to be in $\operatorname{Int}(F)$. For this purpose, we first introduce a function $\xi$, by means of which we express $F$.

We will prove (see Lemma A.3) that, for all $r \in\left(0, p_{2} / p_{1}\right]$, there exists a unique $\xi(r) \in(0, \infty]$ such that

$$
\int_{0}^{\xi(r)} x^{2} f(x) d x=r \int_{0}^{\xi(r)} x f(x) d x
$$

Moreover, we have $\xi(r)<\infty \Longleftrightarrow r<p_{2} / p_{1}$ and $\int_{0}^{x} u^{2} f(u) d u>r \int_{0}^{x} u f(u) d u \Longleftrightarrow x \in$ $(\xi(r), \infty]$.

We define

$$
W \triangleq\left\{v \in(0, \infty)^{3} \mid v_{1} / v_{2} \geq p_{1} / p_{2}, \quad v_{1} / v_{0} \leq \frac{\int_{0}^{\xi\left(v_{2} / v_{1}\right)} x f(x) d x}{\int_{0}^{\xi\left(v_{2} / v_{1}\right)} f(x) d x}\right\}
$$

Proposition $2.2 \quad$ (i) $F=\left(\mathbb{R}^{+} \times\{0\} \times\{0\}\right) \cup W$.

(ii) $(1, m, \delta) \in W$.

(iii) If $m / \delta>p_{1} / p_{2}$ then $(1, m, \delta) \in \operatorname{Int}(W)$.

The proof is given in the mathematical appendix, Section A.

Remark 2.1 Notice that, from Proposition 2.2 (ii), we always have $m / \delta \geq p_{1} / p_{2}$ and from (iii), we have $(1, m, \delta) \in \operatorname{Int}(\mathrm{F})$ provided that $m / \delta>p_{1} / p_{2}$. Therefore, when the 
value of $(P)$ is finite, it follows from Proposition 2.1 that the inequality $m / \delta>p_{1} / p_{2}$ implies that $\operatorname{val}(P)=\operatorname{val}(D)$ and $\operatorname{Sol}(D)$ is non-empty and bounded.

The introduction of the dual problem is justified by the following equivalent formulation of problem $(D)$ which allows us to determine the equilibrium bound in the case of the call option.

Proposition 2.3 The value and the set of solutions to $(D)$ coincide respectively with the value and the set of solutions to the following problem

$$
\begin{aligned}
& \inf _{\lambda \in \mathbb{R}^{3}} \lambda_{0}+\lambda_{1} m+\lambda_{2} \delta \\
& \text { subject to } \int_{0}^{x}\left[\lambda_{0}+\lambda_{1} u+\lambda_{2} u^{2}-\psi(u)\right] f(u) d u \geq 0, \text { for all } x \geq 0 .
\end{aligned}
$$

The proof is given in the mathematical appendix, Section A.

\section{The upper bound determination for the call option}

In this section, we calculate $\operatorname{val}(P)$ in the case of a European call option with strike $K>0$ : in this section we put

$$
\psi(x)=(x-K)^{+}, \text {for all } x \geq 0,
$$

where we use the notation $(x-K)^{+} \triangleq \max \{x-K, 0\}$.

Remark 3.1 Since for all $x \geq 0$, we have $0 \leq \psi(x) \leq x$ and since for all measure $\mu \in \mathcal{C}_{m, \delta}, \int_{0}^{\infty} x f(x) d \mu(x)=m$, we have $\operatorname{val}(\mathrm{P}) \leq m$. The value of problem $(P)$ is therefore finite. In this framework, Proposition 2.1, means that the proposition "val $(P)=\operatorname{val}(D)$ and $\operatorname{Sol}(D)$ is non-empty and bounded" is equivalent to the condition $(1, m, \delta) \in \operatorname{Int}(F)$.

We start with considering the case where $m / \delta=p_{1} / p_{2}$.

Theorem 3.1 If $m / \delta=p_{1} / p_{2}$ then the set $\operatorname{Sol}(D)$ is non-empty, we have

$$
\operatorname{val}(P)=\operatorname{val}(D)=\left(m / p_{1}\right) \int_{0}^{\infty} \psi(u) f(u) d u
$$


and the measure $\mu$ defined by

$$
d \mu \triangleq\left(1-\left(m / p_{1}\right)\right) d \delta_{0}+\left(m / p_{1}\right) \mathbf{1}_{(0, \infty)} d x
$$

is in $\operatorname{Sol}(P)$.

The proof is given in the mathematical appendix, Section B.

From Remark 2.1, we see that it remains to consider the case where $m / \delta>p_{1} / p_{2}$. In that case, the value of $(D)$ depends on several parameters that we now present. When $m / \delta>p_{1} / p_{2}$, we can consider

$$
\bar{x} \triangleq \xi(\delta / m)
$$

where $\xi$ is defined by (3): it is the unique positive real number satisfying

$$
\int_{0}^{\bar{x}} x^{2} f(x) d x=(\delta / m) \int_{0}^{\bar{x}} x f(x) d x
$$

We introduce another parameter $x_{m}$ which also depends on the risk-neutral moments $m$ and $\delta$ and on the true density $f$. We will prove (see Lemma B.1) that when $m / \delta>p_{1} / p_{2}$ there exists a unique $x_{m} \in(0, \infty)$ such that

$$
\int_{0}^{x_{m}} x f(x) d x=m \int_{0}^{x_{m}} f(x) d x
$$

Moreover, we have $\int_{0}^{x} u f(u) d u>m \int_{0}^{x} f(u) d u \Longleftrightarrow x \in\left(x_{m}, \infty\right]$ and $\bar{x}>x_{m}$.

We are now in position to provide the result for the case where $m / \delta>p_{1} / p_{2}$. Since, from Remark 3.1, the value of $(P)$ is finite, we know by Remark 2.1, that $(P)$ and $(D)$ are in strong duality and existence holds for the dual problem. For sake of simplicity, we use the following notation

$$
I(x) \triangleq \int_{0}^{x} f(u) d u, M(x) \triangleq \int_{0}^{x} u f(u) d u, \Delta(x) \triangleq \int_{0}^{x} u^{2} f(u) d u, x \geq 0
$$

Let us also write $d(\bar{x}) \triangleq \bar{x}^{2} \int_{0}^{\bar{x}} \psi(u) f(u) d u-\psi(\bar{x}) \int_{0}^{\bar{x}} u^{2} f(u) d u$. 
Theorem 3.2 Let us assume that $m / \delta>p_{1} / p_{2}$.

(i) If $d(\bar{x})>0$ or if $d(\bar{x})=0$ and $\bar{x}>K$ then

$$
\operatorname{val}(P)=\operatorname{val}(D)=\left(\frac{m}{\int_{0}^{\bar{x}} u f(u) d u}\right) \int_{0}^{\bar{x}} \psi(u) f(u) d u
$$

and the measure $\mu$ defined by

$$
d \mu \triangleq\left(1-\left(\frac{m}{\int_{0}^{\bar{x}} u f(u) d u}\right)\right) d \delta_{0}+\left(\frac{m}{\int_{0}^{\bar{x}} u f(u) d u}\right) \mathbf{1}_{(0, \bar{x})} d x
$$

is in $\operatorname{Sol}(P)$.

(ii) If $d(\bar{x})<0$ or if $d(\bar{x})=0$ and $\bar{x} \leq K$ then there exists $\left(x_{0}, x_{1}\right) \in \mathbb{R}^{+} \times \mathbb{R}^{+}$such that

$$
x_{0} \in\left(0, \min \left\{x_{m}, K\right\}\right) \text { and } x_{1} \in(\max \{\bar{x}, K\}, \infty) \text {, }
$$

$$
\begin{aligned}
& M\left(x_{0}\right) \Delta\left(x_{1}\right)-M\left(x_{1}\right) \Delta\left(x_{0}\right) \\
& \quad=\delta\left[I\left(x_{1}\right) M\left(x_{0}\right)-I\left(x_{0}\right) M\left(x_{1}\right)\right]+m\left[I\left(x_{0}\right) \Delta\left(x_{1}\right)-I\left(x_{1}\right) \Delta\left(x_{0}\right)\right],
\end{aligned}
$$

$$
\begin{aligned}
&\left(x_{0}^{2}-\right.\delta)\left[I\left(x_{1}\right) M\left(x_{0}\right)-I\left(x_{0}\right) M\left(x_{1}\right)\right]+\left(x_{0}-m\right)\left[I\left(x_{0}\right) \Delta\left(x_{1}\right)-I\left(x_{1}\right) \Delta\left(x_{0}\right)\right] \\
&=\left(\int_{0}^{x_{1}} \psi(u) f(u) d u\right)\left(\frac{x_{1}-x_{0}}{\psi\left(x_{1}\right)}\right)\left[\Delta\left(x_{0}\right)-\left(x_{0}+x_{1}\right) M\left(x_{0}\right)+x_{0} x_{1} I\left(x_{0}\right)\right] .
\end{aligned}
$$

We have

$$
\operatorname{val}(P)=\operatorname{val}(D)=\left(\frac{M\left(x_{0}\right)-m I\left(x_{0}\right)}{M\left(x_{0}\right) I\left(x_{1}\right)-I\left(x_{0}\right) M\left(x_{1}\right)}\right) \int_{0}^{x_{1}} \psi(u) f(u) d u
$$

and the measure $\mu$ defined by

$$
d \mu \triangleq\left[\left(\frac{M\left(x_{1}\right)-m I\left(x_{1}\right)}{I\left(x_{0}\right) M\left(x_{1}\right)-I\left(x_{1}\right) M\left(x_{0}\right)}\right) \mathbf{1}_{\left(0, x_{0}\right)}+\left(\frac{M\left(x_{0}\right)-m I\left(x_{0}\right)}{M\left(x_{0}\right) I\left(x_{1}\right)-I\left(x_{0}\right) M\left(x_{1}\right)}\right) \mathbf{1}_{\left(0, x_{1}\right)}\right] d x
$$


is in $\operatorname{Sol}(P)$, for any couple $\left(x_{0}, x_{1}\right) \in \mathbb{R}^{+} \times \mathbb{R}^{+}$which satisfies conditions (8), (9) and (10).

The proof is given in the mathematical appendix, Section B.

Notice that, in light of the proof of Theorem 3.2, it can be seen that the alternative between " $d(\bar{x})>0$ or $d(\bar{x})=0$ and $\bar{x}>K "$ and $" d(\bar{x})<0$ or $d(\bar{x})=0$ and $\bar{x} \leq K "$ corresponds to an alternative concerning the properties of the solutions to problem $(D)$, i.e. according to Proposition 2.3 concerning the solutions to problem (5). Under the first condition, all solutions to problem (5) are such that exactly on constraint is binding. Under the second condition, all solutions are such that exactly two constraints are binding. It can be seen that the first condition amounts to say that $\bar{x}$ is smaller than the smallest positive point for which there exists $\lambda$ satisfying the constraints of problem (5) and such that one exactly of these constraints is binding at this point.

To put an end to this section, we recall the bound on the call option price derived by Levy (1985), Perrakis and Ryan (1984) and Ritchken (1984). In our framework it is given by

$$
B_{P \& R} \triangleq \sup _{\mu \in \mathcal{C}_{m}} \int_{0}^{\infty} \psi(x) f(x) d \mu(x)
$$

where $\mathcal{C}_{m}$ is the set of measures $\mu$ in $\mathcal{C}$ satisfying

$$
\int_{0}^{\infty} f(x) d \mu(x)=1 \text { and } \int_{0}^{\infty} x f(x) d \mu(x)=m
$$

Proposition 3.1 We have

$$
B_{P \& R}=\left(m / p_{1}\right) \int_{0}^{\infty} \psi(x) f(x) d x
$$

The proof is given in the mathematical appendix, Section B. 


\section{Numerical Example}

In this section we observe on some numerical example how the bound of Lo on the call option, i.e.

$$
B_{L o} \triangleq \sup _{\left\{\mathbb{Q} \mid E^{\mathbb{Q}}\left[S_{T}\right]=m, E^{\mathbb{Q}}\left[S_{T}^{2}\right]=\delta\right\}} E^{\mathbb{Q}}\left[\left(S_{T}-K\right)^{+}\right],
$$

can be improved by imposing the equilibrium pricing rule, i.e by considering probability measures that have Radon-Nikodym densities with respect to the true one which decrease with the stock terminal value.

Following some example of Gotoh and Konno (2002), we can report the bound that they obtained by imposing up to fourth moments conditions :

$$
B_{4} \triangleq \sup _{\left\{\mathbb{Q} \mid E^{\mathbb{Q}}\left[S_{T}\right]=m, E^{\mathbb{Q}}\left[S_{T}^{2}\right]=\delta, E^{\mathbb{Q}}\left[S_{T}^{3}\right]=m_{3}, E^{\mathbb{Q}}\left[S_{T}^{4}\right]=m_{4}\right\}} E^{\mathbb{Q}}\left[\left(S_{T}-K\right)^{+}\right]
$$

and thus compare the improvement of Lo's bound entailed by the additional moments conditions to the one entailed by the qualitative restriction on the pricing probability measure.

The example uses the framework of the Black-Scholes model. The market contains one riskfree asset with rate of return $r \geq 0$ and one stock following a log-normal diffusion with drift $\mu \in \mathbb{R}$ and volatility $\sigma \in \mathbb{R}^{*}$. The discounted stock price process $\left(S_{t}\right)_{t \in[0, T]}$ satisfies, for all $t \in[0, T], S_{t}=S_{0} \exp \left\{\left(\mu-r-\sigma^{2} / 2\right) t+\sigma W_{t}\right\}$, and there exists a probability measure $\overline{\mathbb{Q}}$ equivalent to the true one under which $\left(S_{t}\right)_{t \in[0, T]}$ is a martingale. Its RadonNikodym density with respect to the historical probability measure is given by $L_{T}=$ $\exp \left\{-((\mu-r) / 2 \sigma)^{2} T-((\mu-r) / \sigma) W_{T}\right\}$. It is easy to see that

$$
L_{T}=\left(\frac{S_{T}}{S_{0}}\right)^{-\frac{\mu-r}{\sigma^{2}}} \exp \left\{\left(-\frac{\mu-r}{2}+\frac{(\mu-r)^{2}}{2 \sigma^{2}}\right) T\right\} .
$$

The density $L_{T}$ is therefore a nonincreasing function of the stock terminal value if and only the drift $\mu$ is greater than the riskfree rate $r$.

To follow the example presented in Gotoh and Konno, we set the horizon time $T$ to 
$24 / 52$, the riskfree rate to $6 \%$ and the drift $\mu$ to $16 \%$. The stock price at time 0 is fixed to 400 , i.e. $m=S_{0}=400$. We provide the bounds $B_{L o}, B_{4}, B_{P \& R}$ and $\operatorname{val}(P)$ as well as the Black-Scholes price $B S$, for a call option with strike $K$, for several values of the strike $K$. We also let variate the volatility $\sigma$ and hence $\delta$, i.e. the corresponding moment of order 2 under $\overline{\mathbb{Q}}$ of $S_{T}$. We also provide the relative deviation of each bound $B$ from the Black-Scholes price: $e=(B-B S) / B S$.

We observe on table 1 , that in general, $\operatorname{val}(P)$ is much smaller than $B_{4}$. This is false in 2 cases, where the strikes and the volatility are low ( $K=300$ or 350 and $\sigma=20 \%$ ), but the values of $\operatorname{val}(P)$ and $B_{4}$ are very close to each other. Hence, this example shows that when we consider equilibrium pricing probability measures, there is no need to put (unrealistic) additional risk-neutral moments restrictions to improve Lo's bound. The bound that we obtain is very satisfactory since the relative deviation from the BlackScholes price is less than 5\%, expect in 4 cases among 15 where it is between $11 \%$ and $22 \%$. The average relative deviation is about $6 \%$ whereas it is about $24 \%$ for $B_{4}$ and $48 \%$ for $B_{L o}$. Also notice that $B_{P \& R}$ is much smaller than $B_{L o}$. Here again, the equilibrium pricing rule permits to tighten the bound on the call option price (which is given by the current stock price) more significantly than the risk-neutral moment of order 2 restriction.

\section{Here should be inserted Table 1.}

\section{Concluding remarks}

We observe on the numerical example that adding the equilibrium pricing constraints provides, in general, a better bound than the one obtained by adding information on the risk-neutral moments. This encourages us to carry on this work for options with more general payoffs. As it is done by Basso and Pianca (2001) in the case of a finite probability space and without restriction on moments, it would also be of interest to take into account stronger restrictions on preferences such as decreasing absolute risk-aversion, decreasing 
absolute prudence and so on, with or without putting restrictions on moments and in the context of a general probability space.

Also notice that the equilibrium pricing rule can also be valid for a European option expiring at date $t$ lower than the terminal time $T$. Typically, consider an arbitrage-free and complete financial market, with one risky asset $S$, which distributes some dividend $D$. The price at time 0 of a European option with maturity $t$ and payoff $\psi\left(S_{t}\right)$ is given by $E^{\mathbb{Q}}\left[\psi\left(S_{t}\right)\right]=E^{\mathbb{P}}\left[\psi\left(S_{t}\right) M_{t}\right]$, where $M_{t}:=E^{\mathbb{P}}\left[\frac{d \mathbb{Q}}{d \mathbb{P}} \mid \mathcal{F}_{t}\right]$ is the martingale probability measure density with respect to $\mathbb{P}$, conditionally on the information at time $t$. Since the economy is supported by a representative agent, endowed with one unit of the market portfolio, which maximizes some utility of its consumption $c$ and terminal wealth, a necessary condition for equilibrium is that the agent's optimal consumption rate $c_{t}$ is a nonincreasing function of the state price density $M_{t}$ (see e.g. Karatzas 1989). Since at the equilibrium, the consumption process $c_{t}$ must equal the cumulative dividend process $D_{t}$, if we assume that the stock price is an increasing function of this dividend, we obtain that the stock price is a nonincreasing function of the state price density. This last assumption is justified by Jouini and Napp (2003). They show that for a large class of utility functions, there always exist equilibria satisfying this monotonicity condition.

It is possible to derive option prices bounds given other option prices. For example D. Bertsimas and I. Popescu (2002) derived closed form bounds on the price of a European call option, given prices of other options with the same exercise date but different strikes on the same stock. It seems reasonable to assume that, for liquidity reasons, the prices of 1 to 3 near-the-money call options, e.g. with strikes between $70 \%$ and $130 \%$ of the current stock price, are known. Given this information, one can seek for bounds on the equilibrium prices of the call options for other strikes values. This permits to put bounds on the smile, which constitutes a way to separate unrealistic from realistic stochastic volatility models that are used in practice.

Finally, we have set our bounding option prices principle in the case of complete markets in order to use properly the equilibrium condition that provides the decreasing feature of the Radon-Nikodym density of the risk-neutral probability measure with respect to the 
terminal value of the market portfolio. But, under some circumstances, one can argue that in an incomplete market, this latter necessary condition for the pricing probabilities to be compatible with an equilibrium still holds. Of course, in the incomplete market case, the equivalent martingale measure is not unique and there is no reason for the second moment of the underlying asset to be the same under all martingale probability measures. However, one can assume that an upper bound on this second moment under any martingale measure is known. Our bounding principle could then be extended to the incomplete market case, by establishing, for example, that our bound increases with the second moment constraint. This should be the case for the call option and more generally, for derivatives with convex payoffs. 


\section{Mathematical Appendix}

\section{A Proofs of the results stated in Section 2}

In order to shorten and make clear the proofs of Propositions 2.2 and 2.3, we state the five following lemmas. But the reader can directly read the proofs of Propositions 2.2 and 2.3 in Sections A.2 and A.3.

\section{A.1 Technical Lemmas}

The following lemma permits, in particular, to obtain the simple formulation of problem $(D)$ given in Proposition 2.3.

Lemma A.1 Let $h \in L^{1}(0, \infty)$. The following statements are equivalent.

(i) For any function $g$ which is nonnegative and nonincreasing on $(0, \infty)$ and such that $h g \in L^{1}(0, \infty)$, we have $\int_{0}^{x} h(u) g(u) d u \geq 0$, for all $x \geq 0$.

(ii) $\int_{0}^{x} h(u) d u \geq 0$, for all $x \geq 0$.

Proof Let $h \in L^{1}(0, \infty)$. It is clear that (i) implies (ii). Conversely, let us assume that

$$
\int_{0}^{x} h(u) d u \geq 0, \text { for all } x \geq 0
$$

Let $g$ be a function satisfying the requirements of (i) and let $x \in(0, \infty)$. For any $n \in \mathbb{N}^{*}$, consider $\left\{x_{0}, \cdots, x_{n}\right\}$ the regular subdivision of $[0, x]$, with $x_{0}=0$ and $x_{n}=x$. Let us set, for all $u \in[0, x], g_{n}(u) \triangleq \sum_{i=1}^{n} g\left(x_{i}\right) \mathbf{1}_{\left(x_{i-1}, x_{i}\right]}(u)$.

It is easy to see that, if $g$ is continuous at some $u \in(0, x)$ then the sequence $\left(g_{n}(u)\right)_{n}$ converges towards $g(u)$. Since $g$ is nonincreasing, it has a countable number of discontinuities and hence the sequence $\left(g_{n}\right)_{n \in \mathbb{N}^{*}}$ converges to $g$ a.e. on $[0, x]$. One can further check that $0 \leq g_{n} \leq g$ on $[0, x]$, for all $n$. Consequently, the sequence $\left(h g_{n}\right)_{n \in \mathbb{N}^{*}}$ converges to $h g$ a.e. on $[0, x]$ and satisfies: $\left|h g_{n}\right| \leq|h g|$ on $[0, x]$, for all $n$. Since $h g \in L^{1}(0, \infty)$, it 
follows from the dominated convergence theorem that

$$
\int_{0}^{x} h(u) g(u) d u=\lim _{n \rightarrow \infty} \int_{0}^{x} h(u) g_{n}(u) d u
$$

By rewriting $g_{n}$ in the following form $g_{n}=g\left(x_{n}\right) \mathbf{1}_{\left(0, x_{n}\right]}+\sum_{i=1}^{n}\left(g\left(x_{i-1}\right)-g\left(x_{i}\right)\right) \mathbf{1}_{\left(0, x_{i-1}\right]}$ we obtain: $\int_{0}^{x} h(u) g_{n}(u) d u=g\left(x_{n}\right) \int_{0}^{x} h(u) d u+\sum_{i=1}^{n}\left(g\left(x_{i-1}\right)-g\left(x_{i}\right)\right) \int_{0}^{x_{i-1}} h(u) d u$. Since $g$ is nonnegative and nonincreasing on $(0, \infty)$, it then follows from (A.1) that, for all $n$, $\int_{0}^{x} h(u) g_{n}(u) d u \geq 0$. Finally, by (A.2), we have $\int_{0}^{x} h(u) g(u) d u \geq 0$, for all $x \geq 0$. This completes the proof of Lemma A.1.

The following properties of the functions $M / I, \Delta / I$ and $\Delta / M$, where $I, M$ and $\Delta$ are defined in (7), will be used in the sequel. They are easy to obtain by derivation.

Lemma A.2 The functions $x \longmapsto M(x) / I(x), x \longmapsto \Delta(x) / I(x)$ and $x \longmapsto \Delta(x) / M(x)$ are derivable and increasing on $(0, \infty)$.

Now, we prove the existence of the function $\xi$ presented in (3).

Lemma A.3 For all $r \in\left(0, p_{2} / p_{1}\right]$, there exists a unique $\xi(r) \in(0, \infty]$ such that

$$
\int_{0}^{\xi(r)} x^{2} f(x) d x=r \int_{0}^{\xi(r)} x f(x) d x
$$

Moreover $\int_{0}^{x} u^{2} f(u) d u>r \int_{0}^{x} u f(u) d u \Longleftrightarrow x \in(\xi(r), \infty]$, and the function $r \longmapsto \xi(r)$ is continuous on $\left(0, p_{2} / p_{1}\right)$.

Proof Let $r \in\left(0, p_{2} / p_{1}\right]$ and let $\phi$ be the function defined on $\mathbb{R}^{+}$by $\phi(x)=\int_{0}^{x}\left(u^{2}-\right.$ $r u) f(u) d u$. Since $f$ is positive, $\phi$ is decreasing on $(0, r)$ and increasing on $(r, \infty)$. As $\phi$ is continuous and satisfies $\phi(0)=0, \lim _{x \rightarrow \infty} \phi(x)=p_{2}-r p_{1}>0$ when $r<p_{2} / p_{1}$ or $\lim _{x \rightarrow \infty} \phi(x)=0$ when $r=p_{2} / p_{1}$, it follows that there exists a unique $\xi \in(0, \infty]$ such that $\phi<0$ on $(0, \xi), \phi(\xi)=0$ and $\phi>0$ on $(\xi, \infty]$. We clearly have $\xi(r)<\infty \Longleftrightarrow r<p_{2} / p_{1}$.

Noticing that $r=\Delta(\xi(r)) / M(\xi(r))$ for all $r \in\left(0, p_{2} / p_{1}\right)$ and that, by Lemma A.2, the function $\Delta / M$ is continuous and increasing on $(0, \infty)$, we obtain, from the inverse 
function theorem, that $\xi$ is continuous on $\left(0, p_{2} / p_{1}\right)$. This ends the proof of Lemma A.3.

The following technical result is used in the proof of Proposition 2.2.

Lemma A.4 For every $y>0$, there exist $a>0, b \in \mathbb{R}$ and $c>0$ such that

$$
\int_{0}^{x}\left(a+b u+c u^{2}\right) f(u) d u \geq 0 \text { for all } x \geq 0 \text { and } \int_{0}^{y}\left(a+b u+c u^{2}\right) f(u) d u=0 .
$$

Proof Let $y>0$. Let us fix $a>0$. The system

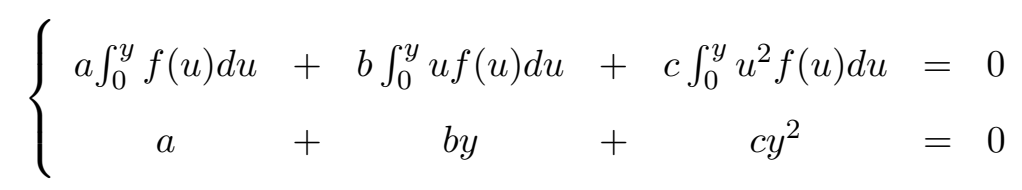

has a solution $(b, c)$ because $y^{2} \int_{0}^{y} u f(u) d u-y \int_{0}^{y} u^{2} f(u) d u>0$. From (A.3) we have

$$
a\left(y \int_{0}^{y} f(u) d u-\int_{0}^{y} u f(u) d u\right)+c\left(y \int_{0}^{y} u^{2} f(u) d u-y^{2} \int_{0}^{y} u f(u) d u\right)=0
$$

and hence $c>0$ because $a>0$ and $y \int_{0}^{y} u^{2} f(u) d u-y^{2} \int_{0}^{y} u f(u) d u<0$. Let us denote by $P$ the function defined on $\mathbb{R}^{+}$by $P(x) \triangleq \int_{0}^{x}\left(a+b u+c u^{2}\right) f(u) d u$. By construction, $P(y)=0$. Let us check that $P(x) \geq 0$ for all $x \geq 0$. Since $P(0)=P(y)=0$ and $f>0$, there exists $z \in(0, y)$, such that $a+b z+c z^{2}=0$. Since $a>0$ and $c>0$, we have $a+b x+c x^{2}>0$ on $[0, z) \cup(y, \infty)$ and $a+b x+c x^{2}<0$ on $(z, y)$. It follows that $P$ is increasing on $[0, z]$ and on $[y, \infty)$ and decreasing on $(z, y)$. Since it satisfies $P(0)=$ $P(y)=0$, this proves that $P(x) \geq 0$, for all $x \geq 0$. This ends the proof of Lemma A.4.

\section{A.2 Proof of Proposition 2.2}

Proof of Proposition 2.2 (i) We prove that $F=\left(\mathbb{R}^{+} \times\{0\} \times\{0\}\right) \cup W$.

Step I. Let us prove that $\left(\mathbb{R}^{+} \times\{0\} \times\{0\}\right) \cup W \subset F$. Let $v \in\left(\mathbb{R}^{+} \times\{0\} \times\{0\}\right) \cup W$ and consider the measure $\mu$ defined by:

$d \mu \triangleq\left(v_{0} / f(0)\right) d \delta_{0}$ if $v \in \mathbb{R}^{+} \times\{0\} \times\{0\}$, 
$d \mu \triangleq\left(v_{0}-v_{1} \frac{\int_{0}^{\xi\left(v_{2} / v_{1}\right)} f(x) d x}{\int_{0}^{\xi\left(v_{2} / v_{1}\right)} x f(x) d x}\right)\left(\frac{1}{f(0)}\right) d \delta_{0}+\left(\frac{v_{1}}{\int_{0}^{\xi\left(v_{2} / v_{1}\right)} x f(x) d x}\right) \mathbf{1}_{\left(0, \xi\left(v_{2} / v_{1}\right)\right)} d x$, if $v \in W$.

One can check that $\mu \in \mathcal{C}$ and $\left(v_{0}, v_{1}, v_{2}\right)=\left(\int_{0}^{\infty} f d \mu, \int_{0}^{\infty} x f d \mu, \int_{0}^{\infty} x^{2} f d \mu\right)$ and hence $v \in F$.

Step II. Let us prove that $F \subset\left(\mathbb{R}^{+} \times\{0\} \times\{0\}\right) \cup W$. Let $v \in F$ and $\mu \in \mathcal{C}$ be such that $v=\left(\int_{0}^{\infty} f d \mu, \int_{0}^{\infty} x f d \mu, \int_{0}^{\infty} x^{2} f d \mu\right)$. By Remark 1.1 there exists $\alpha \in \mathbb{R}^{+}$and $g \in \mathcal{G}$ such that $d \mu=\alpha d \delta_{0}+g d x$. We have:

$$
\left(v_{0}, v_{1}, v_{2}\right)=\left(\alpha f(0)+\int_{0}^{\infty} f(x) g(x) d x, \int_{0}^{\infty} x f(x) g(x) d x, \int_{0}^{\infty} x^{2} f(x) g(x) d x\right) .
$$

Let us denote by $|\{g>0\}|$ the Lebesgue measure of $\{g>0\}$. If $|\{g>0\}|=0$ then $g=0$ a.e. and hence, $v=(\alpha f(0), 0,0) \in \mathbb{R}^{+} \times\{0\} \times\{0\}$.

Let us now consider the case where $|\{g>0\}|>0$. In that case, it is clear that $v \in(0, \infty)^{3}$. Let us prove that

$$
v_{1} / v_{2} \geq p_{1} / p_{2}
$$

Consider the function $h$ defined on $(0, \infty)$ by $h(x) \triangleq x\left(p_{2} / p_{1}-x\right) f(x)$. By construction, $\int_{0}^{\infty} h(x) d x=0$ and since $f$ is positive, the function $x \longmapsto \int_{0}^{x} h(u) d u$ is increasing on $\left(0, p_{2} / p_{1}\right)$ and decreasing on $\left(p_{2} / p_{1}, \infty\right)$. It follows that $\int_{0}^{x} h(u) d u \geq 0$, for all $x \geq 0$. Then, by Lemma A.1, we have $\int_{0}^{x} h(u) g(u) d u \geq 0$, for all $x \geq 0$ and hence, by letting $x$ tend to $\infty,\left(p_{2} / p_{1}\right) v_{1}-v_{2} \geq 0$. We have proved (A.5).

Let us prove that $v_{1} / v_{0} \leq \frac{\int_{0}^{\xi\left(v_{2} / v_{1}\right)} x f(x) d x}{\int_{0}^{\xi\left(v_{2} / v_{1}\right)} f(x) d x}$. When $v_{2} / v_{1}=p_{2} / p_{1}$, since $\xi\left(p_{2} / p_{1}\right)=\infty$, this amounts to prove that

$$
v_{1} / v_{0} \leq p_{1}
$$

As above, we can apply Lemma A.1 to the function $h_{1}$ defined on $(0, \infty)$ by $h_{1}(x)=$ $\left(p_{1}-x\right) f(x)$ and to the function $g$ in order to obtain that $\int_{0}^{x} h_{1}(u) g(u) d u \geq 0$ for all $x \geq 0$ and hence, by passing to the limit when $x$ tend to $\infty, p_{1}\left(v_{0}-\alpha f(0)\right)-v_{1} \geq 0$. Since $\alpha f(0) \geq 0$, that proves (A.6).

From (A.5) we know that, when $|\{g>0\}|>0$ we always have $v_{1} / v_{2} \geq p_{1} / p_{2}$. That proves that, when $v_{1} / v_{2}=p_{1} / p_{2}$, we have $v \in W$. It remains to prove that it is also true 
when $v_{1} / v_{2}>p_{1} / p_{2}$. So, we assume that $v_{1} / v_{2}>p_{1} / p_{2}$ and prove that

$$
v_{1} / v_{0} \leq \frac{\int_{0}^{\xi\left(v_{2} / v_{1}\right)} x f(x) d x}{\int_{0}^{\xi\left(v_{2} / v_{1}\right)} f(x) d x}
$$

For sake of readability, we write $\xi=\xi\left(v_{2} / v_{1}\right)$. Since $\xi \in(0, \infty)$, we can consider the real numbers, $a>0, b \in \mathbb{R}$ and $c>0$, given by Lemma A.4, which are such that $\int_{0}^{x}\left(a+b u+c u^{2}\right) f(u) d u \geq 0$, for all $x \geq 0$ and $\int_{0}^{\xi}\left(a+b u+c u^{2}\right) f(u) d u=0$. Recall that, by Lemma A.3, we have $\int_{0}^{\xi} x^{2} f(x) d x=\left(v_{2} / v_{1}\right) \int_{0}^{\xi} x f(x) d x$. Therefore

$$
\int_{0}^{\xi}\left(a+b u+c u^{2}\right) f(u) d u=\left(\frac{\int_{0}^{\xi} x f(x) d x}{v_{1}}\right)\left(a \frac{v_{1} \int_{0}^{\xi} f(x) d x}{\int_{0}^{\xi} x f(x) d x}+b v_{1}+c v_{2}\right)
$$

and hence

$$
a \frac{v_{1} \int_{0}^{\xi} f(x) d x}{\int_{0}^{\xi} x f(x) d x}+b v_{1}+c v_{2}=0 .
$$

We now show that $a v_{0}+b v_{1}+c v_{2} \geq 0$. With (A.8), this will prove (A.7).

We have $\int_{0}^{x}\left(a+b u+c u^{2}\right) f(u) d u \geq 0$, for all $x \geq 0$. Therefore, by Lemma A.1, we have $\int_{0}^{x}\left(a+b u+c u^{2}\right) f(u) g(u) d u \geq 0$ for all $x \geq 0$ and hence, by letting $x$ tend to $\infty$, $a\left(v_{0}-\alpha f(0)\right)+b v_{1}+c v_{2} \geq 0$. Since $a>0$ and $\alpha f(0) \geq 0$, it follows that $a v_{0}+b v_{1}+c v_{2} \geq 0$. We have obtained that if $v_{1} / v_{2}>p_{1} / p_{2}$ then $v \in W$.

Finally we proved that $F \subset\left(\mathbb{R}^{+} \times\{0\} \times\{0\}\right) \cup W$. This completes Step II and hence proves Proposition 2.2 (i).

Proof of Proposition 2.2 (ii) By definition of $\bar{g}$ (see 2), we have

$$
(1, m, \delta)=\left(\int_{0}^{\infty} f(x) \bar{g}(x) d x, \int_{0}^{\infty} x f(x) \bar{g}(x) d x, \int_{0}^{\infty} x^{2} f(x) \bar{g}(x) d x\right)
$$

and $\bar{g}$ is positive and nonincreasing on $(0, \infty)$. Hence $(1, m, \delta) \in F \backslash\left\{\mathbb{R}^{+} \times\{0\} \times\{0\}\right\}$, i.e. $(1, m, \delta) \in W$.

Proof of Proposition 2.2 (iii) Let us prove that when $m / \delta>p_{1} / p_{2}$, we have $(1, m, \delta) \in$ 
$\operatorname{Int}(W)$. We show that $m<\frac{\int_{0}^{\xi(\delta / m)} x f(x) d x}{\int_{0}^{\xi(\delta / m)} f(x) d x}$. Since $\xi(\delta / m) \in(0, \infty)$, from Lemma A.4, there exists $a^{\prime}>0, b^{\prime} \in \mathbb{R}, c^{\prime}>0$ such that we have

$$
\int_{0}^{x}\left(a^{\prime}+b^{\prime} u+c^{\prime} u^{2}\right) f(u) d u \geq 0, x \geq 0 \text { and } \int_{0}^{\xi(\delta / m)}\left(a^{\prime}+b^{\prime} u+c^{\prime} u^{2}\right) f(u) d u=0 .
$$

Then by Lemma A.1, we have $\int_{0}^{x}\left(a^{\prime}+b^{\prime} u+c^{\prime} u^{2}\right) f(u) \bar{g}(u) d u \geq 0$, for all $x \geq 0$. Since $f>0$ and $\bar{g}>0$ on $(0, \infty)$ and $c^{\prime}>0$, the function $x \longmapsto \int_{0}^{x}\left(a^{\prime}+b^{\prime} u+c^{\prime} u^{2}\right) f(u) \bar{g}(u) d u$ is increasing on $[M, \infty)$ for some large $M$. Hence, from the above inequalities, we deduce that: $\int_{0}^{\infty}\left(a^{\prime}+b^{\prime} u+c^{\prime} u^{2}\right) f(u) \bar{g}(u) d u>0$ and hence $a^{\prime}+b^{\prime} m+c^{\prime} \delta>0$. Now, using the fact that $\int_{0}^{\xi(\delta / m)} x^{2} f(x) d x=(\delta / m) \int_{0}^{\xi(\delta / m)} x f(x) d x$, we deduce from (A.9) that

$$
a^{\prime} m \frac{\int_{0}^{\xi(\delta / m)} f(x) d x}{\int_{0}^{\xi(\delta / m)} x f(x) d x}+b^{\prime} m+c^{\prime} \delta=0 .
$$

Then, since $a^{\prime}>0$, it follows that $m<\frac{\int_{0}^{\xi(\delta / m)} x f(x) d x}{\int_{0}^{\xi(\delta / m)} f(x) d x}$. Thus $(1, m, \delta)$ is in the following subset of $W$ :

$$
O \triangleq\left\{v \in(0, \infty)^{3} \mid v_{1} / v_{2}>p_{1} / p_{2}, \quad v_{1} / v_{0}<\frac{\int_{0}^{\xi\left(v_{2} / v_{1}\right)} x f(x) d x}{\int_{0}^{\xi\left(v_{2} / v_{1}\right)} f(x) d x}\right\}
$$

From Lemma A.3, the function $\xi$ is continuous on $\left(0, p_{2} / p_{1}\right)$ and takes values in $(0, \infty)$. Therefore $O$ is an open set and $(1, m, \delta) \in \operatorname{Int}(W)$. The proof of Proposition 2.2 is completed.

\section{A.3 Proof of Proposition 2.3}

Let us prove that the value and the set of solutions to problem $(D)$ coincide respectively with the value and the set of solutions to the following problem:

$$
\begin{aligned}
& \min _{\lambda \in \mathbb{R}^{3}} \lambda_{0}+\lambda_{1} m+\lambda_{2} \delta \\
& \text { subject to } \int_{0}^{x}\left[\lambda_{0}+\lambda_{1} u+\lambda_{2} u^{2}-\psi(u)\right] f(u) d u \geq 0, \text { for all } x \geq 0 .
\end{aligned}
$$


It suffices to check that, for all $\lambda \in \mathbb{R}^{3}$, the following statements are equivalent.

$$
\begin{gathered}
\lambda_{0} f+\lambda_{1} x f+\lambda_{2} x^{2} f-\psi f \in \mathcal{C}^{*} \\
\int_{0}^{x}\left[\lambda_{0}+\lambda_{1} u+\lambda_{2} u^{2}-\psi(u)\right] f(u) d u \geq 0, \text { for all } x \geq 0 .
\end{gathered}
$$

Let $\lambda \in \mathbb{R}^{3}$. (A.10) holds if and only if $\int_{0}^{\infty}\left[\lambda_{0}+\lambda_{1} x+\lambda_{2} x^{2}-\psi(x)\right] f(x) d \mu(x) \geq 0$ for all $\mu \in \mathcal{C}$. By Remark 1.1, this amounts to the condition

$\alpha\left(\lambda_{0}-\psi(0)\right) f(0)+\int_{0}^{\infty}\left[\lambda_{0}+\lambda_{1} x+\lambda_{2} x^{2}-\psi(x)\right] f(x) g(x) d x \geq 0$, for all $\alpha \in \mathbb{R}^{+}, g \in \mathcal{G}$.

But, $f(0)>0$ and $\psi(0)=0$. It follows that (A.10) holds if and only

$$
\text { a) } \lambda_{0} \geq 0 \text {, b) } \int_{0}^{\infty}\left[\lambda_{0}+\lambda_{1} x+\lambda_{2} x^{2}-\psi(x)\right] f(x) g(x) d x \geq 0 \text {. }
$$

Since by assumption the functions $\psi f, f, x f$ and $x^{2} f$ are in $L^{1}(0, \infty)$, it is clear that $\mathcal{G}$ contains the set $\left\{\mathbf{1}_{(0, x)}, x>0\right\}$. Hence, in (A.12), b) implies a). It follows that (A.12) implies (A.11). Conversely, let us assume that (A.11) holds. Let $g \in \mathcal{G}$. Then, from Lemma A.1, we have (A.12). We have therefore obtained that the conditions (A.10) and (A.11) are equivalent. This ends the proof of Proposition 2.3.

\section{B Proofs of the results stated in Section 3}

In this section, we solve problem $(P)$ in the case of the call option. For this purpose, we use problem $(D)$. For sake of simplicity we introduce the following notation. For $\lambda \in \mathbb{R}^{3}$, we denote by $G^{\lambda}$ the function defined on $\mathbb{R}^{+}$by

$$
G^{\lambda}(x) \triangleq \int_{0}^{x}\left[\lambda_{0}+\lambda_{1} u+\lambda_{2} u^{2}-\psi(u)\right] f(u) d u, \text { for all } x \geq 0
$$

and we set

$$
\mathcal{A} \triangleq\left\{\lambda \in \mathbb{R}^{3} \mid G^{\lambda}(x) \geq 0, \forall x \geq 0\right\}
$$


With this notation and Proposition 2.3, we know that problem $(D)$ can be formulated as follows

$$
\min _{\lambda \in \mathcal{A}} \lambda_{0}+\lambda_{1} m+\lambda_{2} \delta
$$

In the sequel, we will work only with this formulation of problem $(D)$.

The proof of Theorem 3.2 relies on the study of the binding constraints of problem $(D)$. So, we introduce a notation for the set of positive real numbers where some of the constraints $\left\{G^{\lambda}(x) \geq 0, x>0\right\}$ are binding. For $\lambda \in \mathbb{R}^{3}$, we set

$$
\operatorname{bind}(\lambda) \triangleq\left\{x \in(0, \infty) \mid G^{\lambda}(x)=0\right\}
$$

As in the previous section, we begin with stating some lemmas that allow us to shorten the proofs of the main results (Theorems 3.1 and 3.2). But the reader can go directly to the proofs of the theorems in Sections B.2 and B.3.

\section{B.1 Technical Lemmas}

We first show that the parameter $x_{m}$ introduced before the statement of Theorem 3.1 is well defined.

Lemma B.1 Let us assume that $m / \delta>p_{1} / p_{2}$. Then, there exists a unique $x_{m} \in(0, \infty)$ such that

$$
\int_{0}^{x_{m}} x f(x) d x=m \int_{0}^{x_{m}} f(x) d x
$$

and we have $\int_{0}^{x} u f(u) d u>m \int_{0}^{x} f(u) d u \Longleftrightarrow x \in\left(x_{m}, \infty\right]$. Moreover $\bar{x}>x_{m}$ where we recall that $\bar{x} \triangleq \xi(\delta / m)$.

Proof We begin with proving that $m<p_{1}$. Since $m / \delta>p_{1} / p_{2}$, from Proposition 2.2 (iii) we know that $(1, m, \delta) \in \operatorname{Int}(W)$ and hence that $m<\frac{\int_{0}^{\bar{x}} x f(x) d x}{\int_{0}^{\bar{x}} f(x) d x}$, i.e. $m<M(\bar{x}) / I(\bar{x})$. From Lemma A.2, the function $M / I$ is increasing on $(0, \infty)$. Hence we have $m<\frac{\int_{0}^{\infty} x f(x) d x}{\int_{0}^{\infty} f(x) d x}$, i.e. $m<p_{1}$.

Let us consider the function $\phi$ defined on $\mathbb{R}^{+}$by $\phi(x) \triangleq \int_{0}^{x}(u-m) f(u) d u$. The function $\phi$ is continuous on $\mathbb{R}^{+}$. It is decreasing on $(0, m)$, increasing on $(m, \infty)$ and 
satisfies: $\phi(0)=0$ and $\lim _{x \rightarrow \infty} \phi(x)=p_{1}-m>0$. It follows that there exists a unique $x_{m} \in(0, \infty)$ such that $\phi<0$ on $\left(0, x_{m}\right), \phi\left(x_{m}\right)=0$ and $\phi>0$ on $\left(x_{m}, \infty\right]$. Finally, since $m<\frac{\int_{0}^{\bar{x}} x f(x) d x}{\int_{0}^{x} f(x) d x}$ we have $\bar{x}>x_{m}$. This completes the proof of Lemma B.1.

We now state some basic properties of the sets $\mathcal{A}$ and $\operatorname{bind}(\lambda)$ for $\lambda \in \mathcal{A}$.

Lemma B.2 (o) $\mathcal{A} \subset \mathbb{R}^{+} \times \mathbb{R}^{2}$.

(i) Let $\lambda \in \mathcal{A}$. The set $\operatorname{bind}(\lambda)$ has at most two elements.

(ii) Let $\lambda \in \mathcal{A}$. If $\lambda_{2} \leq 0$ then $\operatorname{bind}(\lambda)=\emptyset$.

(iii) Let $\lambda \in \mathcal{A}$. If $\lambda_{2}>0$ then $\lim _{x \rightarrow \infty} G^{\lambda}(x)>0$.

(iv) Let $\lambda \in \mathcal{A}$. If $\operatorname{bind}(\lambda)=\left\{x_{0}, x_{1}\right\}$ with $x_{0}<x_{1}$ then $\lambda_{0}>0, \lambda_{1}<0, \lambda_{2}>0$ and $x_{0}<K<x_{1}$.

Conversely, let $\lambda \in \mathbb{R}^{3}$. If $\lambda_{0}>0, \lambda_{1}<0, \lambda_{2}>0$ and $\operatorname{bind}(\lambda)=\left\{x_{0}, x_{1}\right\}$ with $0<x_{0}<x_{1}$ and $G^{\lambda^{\prime}}\left(x_{0}\right)=G^{\lambda^{\prime}}\left(x_{1}\right)=0$ then $\lambda \in \mathcal{A}$.

The proof of the lemma is essentially based on the fact that, for $\lambda \in \mathcal{A}$, the set $\operatorname{bind}(\lambda)$ is included in the set of $G^{\lambda}$ 's minima and hence, since $f$ is positive, in the set of the points where the parabola $x \longmapsto \lambda_{0}+\lambda_{1} x+\lambda_{2} x^{2}$ intersects the graph of $x \longmapsto \psi(x)=(x-K)^{+}$. Since it is quite long but basic, the proof is omitted. One can have a good intuition on these results and their proofs with a graphical study of the possible intersections of the parabola and the call payoff.

Lemma B.3 Let us assume that $m / \delta>p_{1} / p_{2}$. If $\lambda$ is a solution to problem $(D)$ then the set $\operatorname{bind}(\lambda)$ is non-empty.

Proof Let $\lambda$ be a solution to problem $(D)$. We assume that $\operatorname{bind}(\lambda)=\emptyset$ and obtain a contradiction with the optimal feature of $\lambda$. By assumption, we have $G^{\lambda}(x)>0$, for all $x>0$. Since $m / \delta \neq p_{1} / p_{2}$, there exist $a, b \in \mathbb{R}$ such that

$$
1+a m+b \delta<0 \text { and } 1+a p_{1}+b p_{2}>0 .
$$


For all $\varepsilon>0$, by setting $\lambda_{0}^{\varepsilon} \triangleq \lambda_{0}+\varepsilon, \lambda_{1}^{\varepsilon} \triangleq \lambda_{1}+\varepsilon a$ and $\lambda_{2}^{\varepsilon} \triangleq \lambda_{2}+\varepsilon b$, we have: $\lambda_{0}^{\varepsilon}+\lambda_{1}^{\varepsilon} m+\lambda_{2}^{\varepsilon} \delta<\lambda_{0}+\lambda_{1} m+\lambda_{2} \delta$.

Let us prove that there exists $\varepsilon>0$ such that $\lambda^{\varepsilon} \triangleq\left(\lambda_{0}^{\varepsilon}, \lambda_{1}^{\varepsilon}, \lambda_{2}^{\varepsilon}\right) \in \mathcal{A}$. We write $G^{\varepsilon} \triangleq G^{\lambda^{\varepsilon}}$. By construction we have $G^{\varepsilon}(x)=G^{\lambda}(x)+\varepsilon H(x)$ where

$$
H(x) \triangleq \int_{0}^{x}\left(1+a u+b u^{2}\right) f(u) d u
$$

Since $f$ is positive and since, from the second row of system (B.3), $\lim _{x \rightarrow \infty} H(x)=$ $\left(1+a p_{1}+b p_{2}\right)>0$, there exists $\eta>0$ and $X \geq \eta$ such that $H \geq 0$ on $[0, \eta] \cup[X, \infty)$. Since $G^{\lambda}$ is nonnegative, this implies that for all $\varepsilon>0$,

$$
G^{\varepsilon} \geq 0 \quad \text { on } \quad[0, \eta] \cup[X, \infty)
$$

Since $G^{\lambda}$ is continuous and positive on $(0, \infty)$, it is bounded from below by some constant $M>0$ on $[\eta, X]$. Since the function $H$ is continuous, and thus bounded on $[\eta, X]$, it follows that there exists $\varepsilon>0$ such that, for all $x \in[\eta, X], G^{\varepsilon}(x)=G^{\lambda}(x)+\varepsilon H(x) \geq M+\varepsilon H(x)$. This last inequality together with (B.4) prove that $\lambda^{\varepsilon}$ is in $\mathcal{A}$ and achieve the proof of Lemma B.3.

From Lemmas B.2 (i) and B.3, we know that, at the optimum for problem $(D)$, there exists at least one and at most two positive real numbers where some constraints are binding. In the following lemma, we provide a necessary condition on the value of problem $(D)$ under which a solution $\lambda$ is such that exactly one constraint is binding at some positive real number.

Lemma B.4 Let us assume that $m / \delta>p_{1} / p_{2}$. Let $\lambda$ be a solution to problem $(D)$ such that $\operatorname{bind}(\lambda)=\{y\}$. Then

$$
\lambda_{0}=0, y=\bar{x} \text { and } \operatorname{val}(D)=\lambda_{1} m+\lambda_{2} \delta=\left(\frac{m}{\int_{0}^{\bar{x}} u f(u) d u}\right) \int_{0}^{\bar{x}} \psi(u) f(u) d u
$$


Proof We start with proving that $\lambda_{0}=0$. Assume for the moment that the following result holds: if $\lambda_{0}>0$, then, for all $(a, b, c) \in \mathbb{R}^{3}$,

$$
a \int_{0}^{y} f(u) d u+b \int_{0}^{y} u f(u) d u+c \int_{0}^{y} u^{2} f(u) d u>0 \Longrightarrow a+b m+c \delta \geq 0
$$

Thus, if $\lambda_{0}>0$ then the vectors $(1, m, \delta)$ and $\left(\int_{0}^{y} f(u) d u, \int_{0}^{y} u f(u) d u, \int_{0}^{y} u^{2} f(u)\right)$ are linearly dependent. In other words $\int_{0}^{y} u f(u) d u=m \int_{0}^{y} f(u) d u$ and $\int_{0}^{y} u^{2} f(u) d u=\delta \int_{0}^{y} f(u) d u$. From Lemma B.1, the first equality means that $y=x_{m}$. By definition of $\bar{x}$ (see (6)) and from Lemma A.3, the combination of the two equalities implies that $y=\bar{x}$. This is a contradiction because, by Lemma B.1 again we have, $\bar{x}>x_{m}$. Hence $\lambda_{0}=0$.

We now prove the result that we have assumed above i.e. if $\lambda_{0}>0$ then (B.5) holds for all $(a, b, c) \in \mathbb{R}^{3}$.

Let $(a, b, c) \in \mathbb{R}^{3}$ be such that

$$
a \int_{0}^{y} f(u) d u+b \int_{0}^{y} u f(u) d u+c \int_{0}^{y} u^{2} f(u) d u>0
$$

Let us prove that there exists $\varepsilon>0$ such that $\lambda+(\varepsilon a, \varepsilon b, \varepsilon c) \in \mathcal{A}$. Since $\lambda$ is a solution to problem $(D)$, it will follow that

$$
\lambda_{0}+\lambda_{1} m+\lambda_{2} \delta \leq\left(\lambda_{0}+\varepsilon a\right)+\left(\lambda_{1}+\varepsilon b\right) m+\left(\lambda_{2}+\varepsilon c\right) \delta
$$

i.e. $a+b m+c \delta \geq 0$ and hence, (B.5) will be proved.

Let $\varepsilon>0$. For simplicity, we write $G^{\varepsilon} \triangleq G^{\lambda+\varepsilon(a, b, c)}$. We have

$$
G^{\varepsilon}(x)=\int_{0}^{x}\left[\left(\lambda_{0}+\varepsilon a\right)+\left(\lambda_{1}+\varepsilon b\right) u+\left(\lambda_{2}+\varepsilon c\right) u^{2}-(u-K)^{+}\right] f(u) d u, \text { for all } x \geq 0 .
$$

Since $\lambda_{0}>0$, there exists $\varepsilon_{0}>0$ such that for all $\varepsilon \in\left[0, \varepsilon_{0}\right], \lambda_{0}+\varepsilon a \geq \lambda_{0} / 2>0$. Since $f$ is positive, it follows that there exists $\eta>0$ such that, for all $\varepsilon \in\left[0, \varepsilon_{0}\right]$,

$$
G^{\varepsilon} \geq 0 \text { on }[0, \eta)
$$


Besides we have, for all $x \geq 0, G^{\varepsilon}(x)=G^{\lambda}(x)+\varepsilon H(x)$ where $H$ is defined by

$$
H(x) \triangleq a \int_{0}^{x} f(u) d u+b \int_{0}^{x} u f(u) d u+c \int_{0}^{x} u^{2} f(u) d u
$$

From (B.6), there exists a neighborhood $(\alpha, \beta)$ of $y$ where $H>0$. It follows that, for all $\varepsilon>0$ and for all $x \in(\alpha, \beta)$,

$$
G^{\varepsilon}(x) \geq G^{\lambda}(x) \geq 0
$$

Since $\operatorname{bind}(\lambda) \neq \emptyset$, from Lemma B.2 (ii), we have $\lambda_{2}>0$ and then by Lemma B.2 (iii), $\lim _{x \rightarrow \infty} G^{\lambda}(x)>0$. Hence $G^{\lambda}>0$ on $(0, \infty] \backslash\{y\}$. As it is continuous, it is therefore bounded from below by some positive constant on $[\eta, \alpha] \cup[\beta, \infty]$. Since the functions $f$, $x f$ and $x^{2} f$ are in $L^{1}(0, \infty)$, the function $H$ is bounded. Thus there exists $\varepsilon \in\left(0, \varepsilon_{0}\right)$ such that for all $x \in[\eta, \alpha] \cup[\beta, \infty)$,

$$
G^{\varepsilon}(x)=G^{\lambda}(x)+\varepsilon H(x) \geq 0 .
$$

It follows from (B.7), (B.8) and (B.9) that $G^{\varepsilon} \geq 0$ on $\mathbb{R}^{+}$, i.e. $\lambda+\varepsilon(a, b, c) \in \mathcal{A}$. This ends the proof of (B.5).

Let us now prove that $y=\bar{x}$ and that

$$
\operatorname{val}(D)=\lambda_{1} m+\lambda_{2} \delta=\left(\frac{m}{\int_{0}^{\bar{x}} u f(u) d u}\right) \int_{0}^{\bar{x}} \psi(u) f(u) d u
$$

Using the same kind of arguments as above, one can deduce from the optimal feature of $\lambda$ that, for all $(a, b, c) \in(0, \infty) \times \mathbb{R}^{2}$, we have

$$
a \int_{0}^{y} f(u) d u+b \int_{0}^{y} u f(u) d u+c \int_{0}^{y} u^{2} f(u) d u>0 \Longrightarrow a+b m+c \delta \geq 0 .
$$

This implies that $y$ satisfies $m \int_{0}^{y} u^{2} f(u) d u=\delta \int_{0}^{y} u f(u) d u$ and hence, by definition of $\bar{x}$, that $y=\bar{x}$. Since $\lambda_{0}=0$, we have

$$
G^{\lambda}(\bar{x})=0 \Leftrightarrow \lambda_{1} \int_{0}^{\bar{x}} u f(u) d u+\lambda_{2} \int_{0}^{\bar{x}} u^{2} f(u) d u=\int_{0}^{\bar{x}} \psi(u) f(u) d u
$$


and then it is easy to see that (B.10) holds. This concludes the proof of Lemma B.4.

We now provide a lower bound for the value of problem $(D)$ in the case where $m / \delta>$ $p_{1} / p_{2}$.

Lemma B.5 If $m / \delta>p_{1} / p_{2}$ then for all $\lambda \in \mathcal{A}$ we have

$$
\lambda_{0}+\lambda_{1} m+\lambda_{2} \delta \geq\left(\frac{m}{\int_{0}^{\bar{x}} u f(u) d u}\right) \int_{0}^{\bar{x}} \psi(u) f(u) d u
$$

with strict inequality when $\lambda_{0}>0$.

Proof Let $\lambda \in \mathcal{A}$. Recall that $\bar{x}$ satisfies $\int_{0}^{\bar{x}} x^{2} f(x) d x=(\delta / m) \int_{0}^{\bar{x}} x f(x) d x$. We therefore have

$\lambda_{0}+\lambda_{1} m+\lambda_{2} \delta=\left(\frac{m}{\int_{0}^{\bar{x}} u f(u) d u}\right)\left(\lambda_{0} \frac{\int_{0}^{\bar{x}} u f(u) d u}{m}+\lambda_{1} \int_{0}^{\bar{x}} u f(u) d u+\lambda_{2} \int_{0}^{\bar{x}} u^{2} f(u) d u\right)$.

But, from Lemma B.1, $\frac{\int_{0}^{\bar{x}} u f(u) d u}{m}>\int_{0}^{\bar{x}} f(u) d u$. Since, from Lemma B.2 (o), $\lambda_{0} \geq 0$, it follows that

$$
\begin{aligned}
\lambda_{0}+\lambda_{1} m+\lambda_{2} \delta & \geq\left(\frac{m}{\int_{0}^{\bar{x}} u f(u) d u}\right)\left(\lambda_{0} \int_{0}^{\bar{x}} f(u) d u+\lambda_{1} \int_{0}^{\bar{x}} u f(u) d u+\lambda_{2} \int_{0}^{\bar{x}} u^{2} f(u) d u\right) \\
& \geq\left(\frac{m}{\int_{0}^{x} u f(u) d u}\right) \int_{0}^{\bar{x}} \psi(u) f(u) d u
\end{aligned}
$$

where the first inequality is strict when $\lambda_{0}>0$ and the second one holds because $\lambda \in \mathcal{A}$. This ends the proof of Lemma B.5.

In the following lemma we give a necessary and sufficient condition for the lower bound, given in Lemma B.5, to be attained in problem $(D)$.

$$
\text { Recall that } d(\bar{x})=\bar{x}^{2} \int_{0}^{\bar{x}} \psi(u) f(u) d u-\psi(\bar{x}) \int_{0}^{\bar{x}} u^{2} f(u) d u \text {. }
$$

Lemma B.6 Assume that $m / \delta>p_{1} / p_{2}$. Then, there exists $\left(\lambda_{1}, \lambda_{2}\right) \in \mathbb{R}^{2}$ which satisfies

$$
\left(0, \lambda_{1}, \lambda_{2}\right) \in \mathcal{A} \text { and } \lambda_{1} m+\lambda_{2} \delta=\left(\frac{m}{\int_{0}^{\bar{x}} u f(u) d u}\right) \int_{0}^{\bar{x}} \psi(u) f(u) d u
$$

if and only if $d(\bar{x})>0$ or $d(\bar{x})=0$ and $\bar{x}>K$. 
Proof Let $\left(\lambda_{1}, \lambda_{2}\right) \in \mathbb{R}^{2}$ and set $\lambda \triangleq\left(0, \lambda_{1}, \lambda_{2}\right)$. Using the fact that $\int_{0}^{\bar{x}} x^{2} f(x) d x=$ $(\delta / m) \int_{0}^{\bar{x}} x f(x) d x$ we obtain the following equivalences

$$
\begin{aligned}
\lambda_{1} m+\lambda_{2} \delta=\left(\frac{m}{\int_{0}^{\bar{x}} u f(u) d u}\right) \int_{0}^{\bar{x}} \psi(u) f(u) d u & \Leftrightarrow \int_{0}^{\bar{x}}\left(\lambda_{1} u+\lambda_{2} u^{2}-\psi(u)\right) f(u) d u=0 \\
& \Leftrightarrow G^{\lambda}(\bar{x})=0 .
\end{aligned}
$$

Since $\lambda \in \mathcal{A} \Leftrightarrow G^{\lambda} \geq 0$ it follows that:

$\lambda \in \mathcal{A}$ and $\lambda_{1} m+\lambda_{2} \delta=\left(\frac{m}{\int_{0}^{x} u f(u) d u}\right) \int_{0}^{\bar{x}} \psi(u) f(u) d u$, if and only if, $\lambda \in \mathcal{A}$ and $\bar{x}$ is minimum of $G^{\lambda}$ with $G^{\lambda}(\bar{x})=0$, which is equivalent to, $\lambda \in \mathcal{A}, G^{\lambda}(\bar{x})=0$ and $G^{\lambda^{\prime}}(\bar{x})=0$.

Consequently, since $f$ is positive, we have the equivalence between the existence of $\left(\lambda_{1}, \lambda_{2}\right) \in \mathbb{R}^{2}$ such that we have

$$
\left(0, \lambda_{1}, \lambda_{2}\right) \in \mathcal{A} \text { and } \lambda_{1} m+\lambda_{2} \delta=\left(\frac{m}{\int_{0}^{\bar{x}} u f(u) d u}\right) \int_{0}^{\bar{x}} \psi(u) f(u) d u
$$

and the existence of a solution $\left(\lambda_{1}, \lambda_{2}\right) \in \mathbb{R}^{2}$ to the system

$$
\left\{\begin{array}{ccccc}
\lambda_{1} \int_{0}^{\bar{x}} u f(u) d u & + & \lambda_{2} \int_{0}^{\bar{x}} u^{2} f(u) d u & = & \int_{0}^{\bar{x}} \psi(u) f(u) d u \\
\lambda_{1} \bar{x} & + & \lambda_{2} \bar{x}^{2} & = & \psi(\bar{x})
\end{array}\right.
$$

which satisfies $\left(0, \lambda_{1}, \lambda_{2}\right) \in \mathcal{A}$.

Since $\bar{x}>0$, the determinant of the system (B.11) is positive and hence the system has a unique solution. Let $\left(\lambda_{1}, \lambda_{2}\right)$ be this solution. In order to conclude it remains to prove that

$$
\left(0, \lambda_{1}, \lambda_{2}\right) \in \mathcal{A} \Longleftrightarrow d(\bar{x})>0 \text { or } d(\bar{x})=0 \text { and } \bar{x}>K
$$

From (B.11), $\left(\lambda_{1}, \lambda_{2}\right)$ satisfies

$$
\begin{aligned}
\lambda_{1}\left(\bar{x}^{2} \int_{0}^{\bar{x}} u f(u) d u-\bar{x} \int_{0}^{\bar{x}} u^{2} f(u) d u\right) & =\bar{x}^{2} \int_{0}^{\bar{x}} \psi(u) f(u) d u-\psi(\bar{x}) \int_{0}^{\bar{x}} u^{2} f(u) d u \\
\lambda_{2}\left(\bar{x} \int_{0}^{\bar{x}} u^{2} f(u) d u-\bar{x}^{2} \int_{0}^{\bar{x}} u f(u) d u\right) & =\bar{x} \int_{0}^{\bar{x}} \psi(u) f(u) d u-\psi(\bar{x}) \int_{0}^{\bar{x}} u f(u) d u .
\end{aligned}
$$


Let us check that when $d(\bar{x})<0$ or $d(\bar{x})=0$ and $\bar{x} \leq K$, we have $\left(0, \lambda_{1}, \lambda_{2}\right) \notin \mathcal{A}$. We have

$$
x^{2} \int_{0}^{x} u f(u) d u-x \int_{0}^{x} u^{2} f(u) d u>0, \text { for all } x>0
$$

Therefore when $d(\bar{x})<0$, by (B.12) we have $\lambda_{1}<0$ and hence $\left(0, \lambda_{1}, \lambda_{2}\right) \notin \mathcal{A}$. Indeed, for small enough $x$ we would have $G^{\left(0, \lambda_{1}, \lambda_{2}\right)}(x)=\int_{0}^{x}\left(\lambda_{1} u+\lambda_{2} u^{2}\right) f(u) d u<0$. In the case where $d(\bar{x})=0$ and $\bar{x} \leq K$, we have $\lambda_{1}=0$ from (B.12) and $\lambda_{2}=0$ from (B.13) and (B.14), hence $\left(0, \lambda_{1}, \lambda_{2}\right)=(0,0,0) \notin \mathcal{A}$.

Now we assume that $d(\bar{x})>0$ or $d(\bar{x})=0$ and $\bar{x}>K$ and prove that $\left(0, \lambda_{1}, \lambda_{2}\right) \in \mathcal{A}$. We first prove that $\lambda_{1} \geq 0$ and $\lambda_{2}>0$. Since, in that case, $d(\bar{x}) \geq 0$, from (B.12) we have $\lambda_{1} \geq 0$. Let us prove that $\lambda_{2}>0$. From (B.14), it suffices to prove that the right-hand term in (B.13) is negative. By construction, if $\bar{x} \leq K$ then $d(\bar{x})=0$. Since here $d(\bar{x})>0$ or $d(\bar{x})=0$ and $\bar{x}>K$, we have in any case $\bar{x}>K$ and thus,

$$
\begin{aligned}
r(\bar{x}) & \triangleq \bar{x} \int_{0}^{\bar{x}} \psi(u) f(u) d u-\psi(\bar{x}) \int_{0}^{\bar{x}} u f(u) d u \\
& =\int_{K}^{\bar{x}}(\bar{x}(u-K)-u(\bar{x}-K)) f(u) d u-(\bar{x}-K) \int_{0}^{K} u f(u) d u \\
& =-K \int_{K}^{\bar{x}}(\bar{x}-u) f(u) d u-(\bar{x}-K) \int_{0}^{K} u f(u) d u \\
& <0
\end{aligned}
$$

This proves that $\lambda_{2}>0$.

We are now in position to prove that $\left(0, \lambda_{1}, \lambda_{2}\right) \in \mathcal{A}$. Let us write $\lambda=\left(0, \lambda_{1}, \lambda_{2}\right)$. Since $\lambda_{1} \geq 0, \lambda_{2}>0$ and $\psi=0$ on $[0, K]$, it is clear that $G^{\lambda} \geq 0$ on $[0, K]$. On $(K, \infty)$, the function $G^{\lambda}$ is piecewise monotone, it is nondecreasing (resp. nonincreasing) on the intervals where the polynomial $p(x)=\lambda_{1} x+\lambda_{2} x^{2}-(x-K)$ is nonnegative (resp nonpositive). Since $\lambda_{1} \geq 0$ and $\lambda_{2}>0$, we have $p(K)=\lambda_{1} K+\lambda_{2} K^{2}>0$ and $\lim _{x \rightarrow \infty} p(x)=\infty$. 
Besides, from the second row of system (B.11), we have $p(\bar{x})=0$. Let us prove that there exists $y \in(K, \bar{x})$ such that $p(y)=0$. Assume to the contrary that $p \neq 0$ on $(K, \bar{x})$. Since $p(K)>0$, we then have $p>0$ on $(K, \bar{x})$ and hence $G^{\lambda}$ is increasing on $(K, \bar{x})$. Since $G^{\lambda}$ is continuous, this contradicts the fact that $G^{\lambda}(K)>0, G^{\lambda}(\bar{x})=0$. So, there exists $y \in(K, \bar{x})$ such that $p(y)=0, p>0$ on $[K, y) \cup(\bar{x}, \infty)$ and $p<0$ on $(y, \bar{x})$. The function $G^{\lambda}$ is therefore increasing on $[K, y)$, decreasing on $(y, \bar{x})$ and increasing on $(\bar{x}, \infty)$. Since $G^{\lambda}(K)>0$ and $G^{\lambda}(\bar{x})=0$, it follows that $G^{\lambda}(x) \geq 0$, for all $x \geq K$. It ensues that $G^{\lambda} \geq 0$ on $\mathbb{R}^{+}$and hence $\lambda \in \mathcal{A}$. This completes the proof of Lemma B.6.

We now provide a necessary condition for a solution $\lambda$ to problem $(D)$ to be such that exactly two constraints are binding at some positive real numbers.

Lemma B.7 Let us assume that $m / \delta>p_{1} / p_{2}$. Let $\lambda$ be a solution to problem $(D)$ such that $\operatorname{bind}(\lambda)=\left\{x_{0}, x_{1}\right\}$ with $x_{0}<x_{1}$. Then there exists $(\alpha, \beta) \in(0, \infty)^{2}$ such that

$$
\begin{aligned}
& \alpha \int_{0}^{x_{0}} f(u) d u+\beta \int_{0}^{x_{1}} f(u) d u=1 \\
& \alpha \int_{0}^{x_{0}} u f(u) d u+\beta \int_{0}^{x_{1}} u f(u) d u=m \\
& \alpha \int_{0}^{x_{0}} u^{2} f(u) d u+\beta \int_{0}^{x_{1}} u^{2} f(u) d u=\delta
\end{aligned}
$$

and we have $\operatorname{val}(D)=\lambda_{0}+\lambda_{1} m+\lambda_{2} \delta=\beta \int_{0}^{x_{1}} \psi(u) f(u) d u$.

Proof Let $\lambda$ be a solution to problem $(D)$ such that $\operatorname{bind}(\lambda)=\left\{x_{0}, x_{1}\right\}$ with $x_{0}<x_{1}$. From Lemma B.2 (iv), we have $x_{0}<K<x_{1}, \lambda_{0}>0, \lambda_{1}<0$ and $\lambda_{2}>0$. Since $\lambda_{0}>0$ and $\lambda_{2}>0$, we can use the same kind of arguments as in the proof of Lemma B.4 in order to deduce from the optimal feature of $\lambda$ that, for all $(a, b, c) \in \mathbb{R}^{3}$, if

$$
\begin{aligned}
& a \int_{0}^{x_{0}} f(u) d u+b \int_{0}^{x_{0}} u f(u) d u+c \int_{0}^{x_{0}} u^{2} f(u) d u>0 \\
& \text { and } \\
& a \int_{0}^{x_{1}} f(u) d u+b \int_{0}^{x_{1}} u f(u) d u+c \int_{0}^{x_{1}} u^{2} f(u) d u>0
\end{aligned}
$$

then $a+b m+c \delta \geq 0$ 
From Farkas Lemma, this implies that there exists $(\alpha, \beta) \in \mathbb{R}^{+2}$ such that

$$
\begin{array}{ll}
\alpha \int_{0}^{x_{0}} f(u) d u & +\beta \int_{0}^{x_{1}} f(u) d u= \\
\alpha \int_{0}^{x_{0}} u f(u) d u+\beta \int_{0}^{x_{1}} u f(u) d u & =m \\
\alpha \int_{0}^{x_{0}} u^{2} f(u) d u+\beta \int_{0}^{x_{1}} u^{2} f(u) d u & =\delta .
\end{array}
$$

We have already remarked, in the proof of Lemma B.4 that for fixed $i$, the vectors $\left(\int_{0}^{x_{i}} f(u) d u, \int_{0}^{x_{i}} u f(u) d u, \int_{0}^{x_{i}} u^{2} f(u) d u\right)$ and $(1, m, \delta)$ can not be linearly dependent. We therefore have $\alpha>0$ and $\beta>0$.

Let us check that $\operatorname{val}(D)=\lambda_{0}+\lambda_{1} m+\lambda_{2} \delta=\beta \int_{0}^{x_{1}} \psi(u) f(u) d u$. From (B.16), the fact that $G^{\lambda}\left(x_{0}\right)=G^{\lambda}\left(x_{1}\right)=0$ and $x_{0}<K$ we obtain

$\operatorname{val}(D)=\lambda_{0}+\lambda_{1} m+\lambda_{2} \delta=\alpha \int_{0}^{x_{0}} \psi(u) f(u) d u+\beta \int_{0}^{x_{1}} \psi(u) f(u) d u=\beta \int_{0}^{x_{1}} \psi(u) f(u) d u$

This ends the proof of Lemma B.7.

Lemma B.8 Let us assume that $m / \delta>p_{1} / p_{2}$. Let $\left(x_{0}, x_{1}\right) \in \mathbb{R}^{2}$ be such that $0<x_{0}<$ $x_{1}$. The system

$$
\begin{aligned}
& \alpha \int_{0}^{x_{0}} f(u) d u+\beta \int_{0}^{x_{1}} f(u) d u=1 \\
& \alpha \int_{0}^{x_{0}} u f(u) d u+\beta \int_{0}^{x_{1}} u f(u) d u=m \\
& \alpha \int_{0}^{x_{0}} u^{2} f(u) d u+\beta \int_{0}^{x_{1}} u^{2} f(u) d u=\delta
\end{aligned}
$$

has a solution $(\alpha, \beta) \in(0, \infty) \times(0, \infty)$ if and only if $x_{0}$ and $x_{1}$ satisfy the following conditions

$$
\begin{gathered}
x_{1} \in(\bar{x}, \infty) x_{0} \in\left(0, x_{m}\right) \text { and } x_{1} \in(\bar{x}, \infty) \\
M\left(x_{0}\right) \Delta\left(x_{1}\right)-M\left(x_{1}\right) \Delta\left(x_{0}\right)=\quad \delta\left[I\left(x_{1}\right) M\left(x_{0}\right)-I\left(x_{0}\right) M\left(x_{1}\right)\right] \\
+m\left[I\left(x_{0}\right) \Delta\left(x_{1}\right)-I\left(x_{1}\right) \Delta\left(x_{0}\right)\right] .
\end{gathered}
$$


Under these conditions, we have

$$
\beta=\frac{M\left(x_{0}\right)-m I\left(x_{0}\right)}{I\left(x_{1}\right) M\left(x_{0}\right)-I\left(x_{0}\right) M\left(x_{1}\right)} .
$$

Proof Let $\left(x_{0}, x_{1}\right) \in \mathbb{R}^{2}$ be such that $0<x_{0}<x_{1}$. We first prove that the system (B.17) has a solution $(\alpha, \beta) \in \mathbb{R}^{2}$ if and only if $x_{0}$ and $x_{1}$ satisfy (B.19). For sake of simplicity, we set $I_{i}=I\left(x_{i}\right), M_{i}=M\left(x_{i}\right)$ and $\Delta_{i}=\Delta\left(x_{i}\right)$, for $i=0,1$. Since $0<x_{0}<x_{1}$ and the functions $M / I$ and $\Delta / M$ are increasing on $(0, \infty)$ (see Lemma A.2), we have

$$
I_{0} M_{1}-I_{1} M_{0}>0 \text { and } M_{0} \Delta_{1}-M_{1} \Delta_{0}>0 .
$$

It follows that the system made of the first (resp. last) two rows of (B.17) has a unique solution $(\bar{\alpha}, \bar{\beta}) \in \mathbb{R}^{2}$ (resp. $\left.(\underline{\alpha}, \beta) \in \mathbb{R}^{2}\right)$. Thus, the system (B.17) has a solution $(\alpha, \beta)$ $\in \mathbb{R}^{2}$ if and only if $(\bar{\alpha}, \bar{\beta})=(\underline{\alpha}, \underline{\beta})$. We have $(\bar{\alpha}, \bar{\beta})=\left(\frac{M_{1}-m I_{1}}{I_{0} M_{1}-I_{1} M_{0}}, \frac{M_{0}-m I_{0}}{I_{1} M_{0}-I_{0} M_{1}}\right)$ and $(\underline{\alpha}, \underline{\beta})=\left(\frac{m \Delta_{1}-\delta M_{1}}{M_{0} \Delta_{1}-M_{1} \Delta_{0}}, \frac{m \Delta_{0}-\delta M_{0}}{M_{1} \Delta_{0}-M_{0} \Delta_{1}}\right)$. One can check that these couples coincide if and only if $x_{0}$ and $x_{1}$ satisfy (B.19). Under this condition, we have

$$
(\alpha, \beta)=\left(\frac{m \Delta_{1}-\delta M_{1}}{M_{0} \Delta_{1}-M_{1} \Delta_{0}}, \frac{M_{0}-m I_{0}}{I_{1} M_{0}-I_{0} M_{1}}\right) .
$$

From (B.20), it then follows that, $(\alpha, \beta)$ is in $(0, \infty)^{2}$ if and only if $m \Delta_{1}-\delta M_{1}>0$ and $M_{0}-m I_{0}<0$. But, from Lemmas A.3 and B.1, we have $m \Delta(x)-\delta M(x)>0 \Leftrightarrow x>\bar{x}$ and $M(x)-m I(x)<0 \Leftrightarrow x<x_{m}$.

Finally, we have obtained that, for $\left(x_{0}, x_{1}\right) \in \mathbb{R}^{2}$ such that $0<x_{0}<x_{1}$, the system (B.17) has a solution $(\alpha, \beta) \in(0, \infty)^{2}$ if and only if $x_{0}$ and $x_{1}$ satisfy (B.19) and $x_{0} \in\left(0, x_{m}\right)$ and $x_{1} \in(\bar{x}, \infty)$. This ends the proof of Lemma B.8.

Lemma B.9 Let $\left(x_{0}, x_{1}\right) \in \mathbb{R}^{2}$ be such that $0<x_{0}<K<x_{1}$. There exists $\lambda \in \mathcal{A}$ such that $\operatorname{bind}(\lambda)=\left\{x_{0}, x_{1}\right\}$ if and only if

$$
\left(\int_{0}^{x_{1}} \psi(u) f(u) d u\right)\left(\left(x_{1}-x_{0}\right) / \psi\left(x_{1}\right)\right)\left[\Delta\left(x_{0}\right)-\left(x_{0}+x_{1}\right) M\left(x_{0}\right)+x_{0} x_{1} I\left(x_{0}\right)\right]
$$




$$
\begin{aligned}
= & x_{0}\left[I\left(x_{0}\right) \Delta\left(x_{1}\right)-\Delta\left(x_{0}\right) I\left(x_{1}\right)\right]+x_{0}^{2}\left[I\left(x_{1}\right) M\left(x_{0}\right)-I\left(x_{0}\right) M\left(x_{1}\right)\right] \\
& +M\left(x_{1}\right) \Delta\left(x_{0}\right)-M\left(x_{0}\right) \Delta\left(x_{1}\right) .
\end{aligned}
$$

Proof Let $\left(x_{0}, x_{1}\right) \mathbb{R}^{2}$ be such that $0<x_{0}<K<x_{1}$. We first prove that the system below has a solution $\lambda \in \mathbb{R}^{3}$ if and only if $\left(x_{0}, x_{1}\right)$ satisfy condition (B.21).

$$
\left\{\begin{array}{llll}
\lambda_{0} & +\lambda_{1} x_{0} & +\lambda_{2} x_{0}^{2} & =0 \\
\lambda_{0} & +\lambda_{1} x_{1} & +\lambda_{2} x_{1}^{2} & =\psi\left(x_{1}\right) \\
\lambda_{0} I\left(x_{0}\right) & +\lambda_{1} M\left(x_{0}\right)+\lambda_{2} \Delta\left(x_{0}\right) & = & 0 \\
\lambda_{0} I\left(x_{1}\right) & +\lambda_{1} M\left(x_{1}\right)+\lambda_{2} \Delta\left(x_{1}\right) & =\int_{0}^{x_{1}} \psi(u) f(u) d u
\end{array} .\right.
$$

Here again, for sake of simplicity, we set $I\left(x_{I}\right)=I_{i}, M\left(x_{i}\right)=M_{i}$ and $\Delta_{i}=\Delta\left(x_{i}\right)$, for $i=0,1$. Let us prove that the system made of the first three rows of (B.22) has a unique solution. Let $d$ be its determinant. We prove that $d>0$. After a few calculations we obtain

$$
d:=\left|\begin{array}{ccc}
1 & x_{0} & x_{0}^{2} \\
1 & x_{1} & x_{1}^{2} \\
I_{0} & M_{0} & \Delta_{0}
\end{array}\right|=\left(x_{1}-x_{0}\right) I_{0}\left[\frac{\Delta_{0}}{I_{0}}-\left(x_{0}+x_{1}\right) \frac{M_{0}}{I_{0}}+x_{1} x_{0}\right]
$$

By Jensen's inequality, we have $\frac{\Delta_{0}}{I_{0}}=\frac{\int_{0}^{x_{0}} u^{2} f(u) d u}{\int_{0}^{x_{0}} f(u) d u} \geq\left(\frac{\int_{0}^{x_{0}} u f(u) d u}{\int_{0}^{x_{0}} f(u) d u}\right)^{2}=\left(\frac{M_{0}}{I_{0}}\right)^{2}$. Hence $\frac{\Delta_{0}}{I_{0}}-\left(x_{0}+x_{1}\right) \frac{M_{0}}{I_{0}}+x_{1} x_{0} \geq\left(\frac{M_{0}}{I_{0}}\right)^{2}-\left(x_{0}+x_{1}\right) \frac{M_{0}}{I_{0}}+x_{1} x_{0}=\left(x_{0}-\frac{M_{0}}{I_{0}}\right)\left(x_{1}-\frac{M_{0}}{I_{0}}\right)$. Since $x_{0}<x_{1}$ and $\frac{M_{0}}{I_{0}}<x_{0}$, it follows that $d>0$. Therefore the system (B.22) has a solution if and only if the solution to the system made of the first 3 equations, that we denote by $\lambda$, is a solution to the fourth. One can obtain $\lambda$ in function of $x_{0}$ and $x_{1}$ as follows

$$
\begin{aligned}
& \lambda_{0}=\frac{x_{0}\left(x_{0} M_{0}-\Delta_{0}\right) \psi\left(x_{1}\right)}{\left(x_{1}-x_{0}\right)\left[\Delta_{0}-\left(x_{0}+x_{1}\right) M_{0}+x_{1} x_{0} I_{0}\right]}, \\
& \lambda_{1}=\frac{\left(\Delta_{0}-x_{0}^{2} I_{0}\right) \psi\left(x_{1}\right)}{\left(x_{1}-x_{0}\right)\left[\Delta_{0}-\left(x_{0}+x_{1}\right) M_{0}+x_{1} x_{0} I_{0}\right]}, \\
& \lambda_{2}=\frac{\left(x_{0} I_{0}-M_{0}\right) \psi\left(x_{1}\right)}{\left(x_{1}-x_{0}\right)\left[\Delta_{0}-\left(x_{0}+x_{1}\right) M_{0}+x_{1} x_{0} I_{0}\right]} .
\end{aligned}
$$


One can check that $\lambda$ satisfies

$$
\lambda_{0} I_{1}+\lambda_{1} M_{1}+\lambda_{2} \Delta_{1}=\int_{0}^{x_{1}} \psi(u) f(u) d u
$$

if and only if $x_{0}$ and $x_{1}$ satisfy (B.21). We therefore have obtained that the system (B.22) has a solution $\lambda \in \mathbb{R}^{3}$ if and only if $x_{0}$ and $x_{1}$ satisfy condition (B.21).

We have remarked that $\left(x_{1}-x_{0}\right)\left[\Delta_{0}-\left(x_{0}+x_{1}\right) M_{0}+x_{1} x_{0} I_{0}\right]>0$. Since $x_{1}>K$, we have $\psi\left(x_{1}\right)>0$ and since $x_{0}>0$, we have $x_{0} M_{0}-\Delta_{0}>0, \Delta_{0}-x_{0}^{2} I_{0}<0$ and $x_{0} I_{0}-M_{0}>0$. Thus, from (B.23), (B.24) and (B.25), when the system (B.22) has a solution $\lambda$, this solution satisfies $\lambda_{0}>0, \lambda_{1}<0$ and $\lambda_{2}>0$.

We are now in position to prove the equivalence stated in the lemma. First notice that, using the fact that $x_{0}<K$, it is easy to see that $\lambda \in \mathbb{R}^{3}$ satisfies (B.22) if and only if $G^{\lambda}\left(x_{0}\right)=G^{\lambda}\left(x_{1}\right)=G^{\lambda^{\prime}}\left(x_{0}\right)=G^{\lambda^{\prime}}\left(x_{1}\right)=0$.

Let us assume that there exists $\lambda \in \mathcal{A}$ such that $\operatorname{bind}(\lambda)=\left\{x_{0}, x_{1}\right\}$. Then $G^{\lambda^{\prime}}\left(x_{0}\right)=$ $G^{\lambda^{\prime}}\left(x_{1}\right)=0$ and thus, from what precedes, $x_{0}$ and $x_{1}$ satisfy (B.21). Conversely, if $x_{0}$ and $x_{1}$ satisfy (B.21) then there exists some $\lambda \in \mathbb{R}^{3}$ which is solution to system (B.22) and such that $\lambda_{0}>0, \lambda_{1}<0$ and $\lambda_{2}>0$. From Lemma B.2 (iv), it follows that $\lambda \in \mathcal{A}$. This ends the proof of Lemma B.9.

\section{B.2 Proof of Theorem 3.1}

Let us assume that $m / \delta=p_{1} / p_{2}$. We first prove that

$$
\operatorname{val}(D) \geq\left(m / p_{1}\right) \int_{0}^{\infty} \psi(u) f(u) d u
$$

Let $\lambda \in \mathcal{A}$. Since $(1, m, \delta)$ is in $F$ and $m / \delta=p_{1} / p_{2}$, by Proposition 2.2 we have $0<$ $m \leq p_{1}$. By Lemma B.2 (o) we know that $\lambda_{0} \geq 0$. Hence we have $\lambda_{0}+\lambda_{1} m+\lambda_{2} \delta \geq$ $\left(m / p_{1}\right)\left(\lambda_{0}+\lambda_{1} p_{1}+\lambda_{2} p_{2}\right)$. We then obtain (B.26) by using the equality $\lambda_{0}+\lambda_{1} p_{1}+\lambda_{2} p_{2}=$ $\int_{0}^{\infty}\left(\lambda_{0}+\lambda_{1} x+\lambda_{2} x^{2}\right) f(x) d x$ and the fact that $\lambda \in \mathcal{A}$ and hence $\int_{0}^{\infty}\left(\lambda_{0}+\lambda_{1} u+\lambda_{2} u^{2}\right) f(u) d u-$ $\int_{0}^{\infty} \psi(u) f(u) d u \geq 0$. 
It remains to prove that the lower bound in (B.26) is attained. Admit for the moment that the function $\Psi$ defined on $\mathbb{R}^{+}$by

$$
\Psi(0)=0 \text { and } \Psi(x)=\frac{\int_{0}^{x} \psi(u) f(u) d u}{\int_{0}^{x} u f(u) d u} \text { for } x>0
$$

is nondecreasing. Then, for $\lambda \triangleq\left(0, \frac{\int_{0}^{\infty} \psi(u) f(u) d u}{p_{1}}, 0\right)$, we have $\lambda_{0}+\lambda_{1} m+\lambda_{2} \delta=$ $\left(m / p_{1}\right) \int_{0}^{\infty} \psi(u) f(u) d u$ and for all $x \geq 0$,

$$
G^{\lambda}(x)=\left(m / p_{1}\right)\left(\int_{0}^{\infty} \psi(u) f(u) d u\right)\left(\int_{0}^{x} u f(u) d u\right)-\int_{0}^{x} \psi(u) f(u) d u \geq 0,
$$

so that $\lambda \in \mathcal{A}$. That proves that the lower bound in (B.26) is attained, i.e. that problem $(D)$ has a solution and its value is given by

$$
\operatorname{val}(D)=\left(m / p_{1}\right) \int_{0}^{\infty} \psi(u) f(u) d u
$$

In order to check that $\operatorname{val}(P)=\operatorname{val}(D)$, one first notice that by construction $\operatorname{val}(P) \leq$ $\operatorname{val}(D)$ and hence $\operatorname{val}(P) \leq\left(m / p_{1}\right) \int_{0}^{\infty} \psi(x) f(x) d x$. One second show that the measure $\mu$ defined by $d \mu \triangleq\left(1-m / p_{1}\right) d \delta_{0}+\left(m / p_{1}\right) \mathbf{1}_{(0, \infty)} d x$ is in $\mathcal{C}_{m, \delta}$ and satisfies $\int_{0}^{\infty} \psi f d \mu=$ $\left(m / p_{1}\right) \int_{0}^{\infty} \psi(u) f(u) d u$.

It remains to prove what we have admitted above, i.e. that the function $\Psi$ is nondecreasing on $\mathbb{R}^{+}$. Using the fact that $f$ is positive, it is easy to check that $\operatorname{sign}\left[\Psi^{\prime}(x)\right]$ $=\operatorname{sign}[-r(x)]$ with $r(x)=x \int_{0}^{x} \psi(u) f(u) d u-\psi(x) \int_{0}^{x} u f(u) d u$, for all $x \in \mathbb{R}^{+}$. We have $r \equiv 0$ on $[0, K]$ and we already saw that $r<0$ on $(K, \infty)$, see (B.15). This proves that $\Psi$ is nondecreasing on $\mathbb{R}^{+}$. The proof of Theorem 3.1 is completed.

\section{B.3 Proof of Theorem 3.2}

We assume that $m / \delta>p_{1} / p_{2}$. We know from Remark 3.1 that the value of problem $(P)$ is finite. We then deduce from Remark 2.1 that strong duality holds between the primal 
and dual problems:

$$
\operatorname{val}(P)=\operatorname{val}(D)
$$

and the dual problem $(D)$ has at least one solution. We can therefore use optimality conditions on some solution to problem $(D)$ in order to prove the theorem.

Proof of Theorem 3.2 (i) Let us assume that $d(\bar{x})>0$ or $d(\bar{x})=0$ and $\bar{x}>K$. Then by Lemma B.6, there exists $\left(\lambda_{1}, \lambda_{2}\right) \in \mathbb{R}^{2}$ such that $\left(0, \lambda_{1}, \lambda_{2}\right) \in \mathcal{A}$ and $\lambda_{1} m+\lambda_{2} \delta=$ $\frac{m}{\int_{0}^{x} u f(u) d u} \int_{0}^{\bar{x}} \psi(u) f(u) d u$. But from Lemma B.5, we know that

$$
\operatorname{val}(D) \geq \frac{m}{\int_{0}^{\bar{x}} u f(u) d u} \int_{0}^{\bar{x}} \psi(u) f(u) d u
$$

With (B.27), it follows that

$$
\operatorname{val}(P)=\operatorname{val}(D)=\frac{m}{\int_{0}^{\bar{x}} u f(u) d u} \int_{0}^{\bar{x}} \psi(u) f(u) d u .
$$

Then, it is easy to see that the measure $\mu$ defined by

$$
d \mu \triangleq\left(1-\left(\frac{m}{\int_{0}^{\bar{x}} u f(u) d u}\right)\right) d \delta_{0}+\left(\frac{m}{\int_{0}^{\bar{x}} u f(u) d u}\right) \mathbf{1}_{(0, \bar{x})} d x
$$

is in $\mathcal{C}_{m, \delta}$ and satisfies $\int_{0}^{\infty} \psi(x) f(x) d \mu(x)=\frac{m}{\int_{0}^{x} u f(u) d u} \int_{0}^{\bar{x}} \psi(u) f(u) d u$. Hence, $\mu \in \operatorname{Sol}(P)$. This ends the proof of Theorem 3.2 (i).

Proof of Theorem 3.2 (ii) We now assume that $d(\bar{x})<0$ or $d(\bar{x})=0$ and $\bar{x} \leq K$. Let $\lambda$ be a solution to problem $(D)$. From Lemmas B.2 (i) and B.3, we know that the set $\operatorname{bind}(\lambda)$ is not empty and has at most two elements. We prove that it contains exactly two elements. Assume to the contrary that $\operatorname{bind}(\lambda)=\{y\}$ for some $y \in(0, \infty)$. Then by Lemma B.4, we have $\lambda_{0}=0, y=\bar{x}$ and $\operatorname{val}(D)=\frac{m}{\int_{0}^{\bar{x}} u f(u) d u} \int_{0}^{\bar{x}} \psi(u) f(u) d u$. So, we have $\lambda=\left(0, \lambda_{1}, \lambda_{2}\right) \in \mathcal{A}$ and $\lambda_{1} m+\lambda_{2} \delta=\frac{m}{\int_{0}^{x} u f(u) d u} \int_{0}^{\bar{x}} \psi(u) f(u) d u$. From Lemma B.6, this can happen only in the case where $d(\bar{x})>0$ or $d(\bar{x})=0$ and $\bar{x}>K$. We conclude that $\operatorname{bind}(\lambda)$ contains exactly two elements.

Let us write $\operatorname{bind}(\lambda)=\left\{x_{0}, x_{1}\right\}$ with $0<x_{0}<x_{1}$. By Lemma B.2 (iv) we have 
$0<x_{0}<K<x_{1}$. Then, from Lemmas B.7 and B.8 we deduce that $x_{0}$ and $x_{1}$ satisfy $x_{0} \in\left(0, \min \left\{x_{m}, K\right\}\right), x_{1} \in(\max \{\bar{x}, K\}, \infty)$ and

$$
\begin{aligned}
M\left(x_{0}\right) \Delta\left(x_{1}\right)-M\left(x_{1}\right) \Delta\left(x_{0}\right)= & \delta\left[I\left(x_{1}\right) M\left(x_{0}\right)-I\left(x_{0}\right) M\left(x_{1}\right)\right] \\
& +m\left[I\left(x_{0}\right) \Delta\left(x_{1}\right)-I\left(x_{1}\right) \Delta\left(x_{0}\right)\right]
\end{aligned}
$$

and that

$$
\operatorname{val}(D)=\lambda_{0}+\lambda_{1} m+\lambda_{2} \delta=\left(\frac{M\left(x_{0}\right)-m I\left(x_{0}\right)}{I\left(x_{0}\right) M\left(x_{1}\right)-I\left(x_{1}\right) M\left(x_{0}\right)}\right) \int_{0}^{x_{1}} \psi(u) f(u) d u .
$$

Finally, by Lemma B.9, $x_{0}$ and $x_{1}$ satisfy

$$
\begin{aligned}
& \left(\int_{0}^{x_{1}} \psi(u) f(u) d u\right)\left(\left(x_{1}-x_{0}\right) / \psi\left(x_{1}\right)\right)\left[\Delta\left(x_{0}\right)-\left(x_{0}+x_{1}\right) M\left(x_{0}\right)+x_{0} x_{1} I\left(x_{0}\right)\right] \\
& =x_{0}\left[I\left(x_{0}\right) \Delta\left(x_{1}\right)-\Delta\left(x_{0}\right) I\left(x_{1}\right)\right]+x_{0}^{2}\left[I\left(x_{1}\right) M\left(x_{0}\right)-I\left(x_{0}\right) M\left(x_{1}\right)\right] \\
& +M\left(x_{1}\right) \Delta\left(x_{0}\right)-M\left(x_{0}\right) \Delta\left(x_{1}\right)
\end{aligned}
$$

i.e. by (B.28),

$$
\begin{aligned}
& \left(\int_{0}^{x_{1}} \psi(u) f(u) d u\right)\left(\left(x_{1}-x_{0}\right) / \psi\left(x_{1}\right)\right)\left[\Delta\left(x_{0}\right)-\left(x_{0}+x_{1}\right) M\left(x_{0}\right)+x_{0} x_{1} I\left(x_{0}\right)\right] \\
& =\left(x_{0}-m\right)\left[I\left(x_{0}\right) \Delta\left(x_{1}\right)-I\left(x_{1}\right) \Delta\left(x_{0}\right)\right]+\left(x_{0}^{2}-\delta\right)\left[I\left(x_{1}\right) M\left(x_{0}\right)-I\left(x_{0}\right) M\left(x_{1}\right)\right] .
\end{aligned}
$$

We just proved that, when $d(\bar{x})<0$ or $d(\bar{x})=0$ and $\bar{x} \leq K$, there exists $\left(x_{0}, x_{1}\right) \in \mathbb{R}^{2}$ which satisfies conditions (8), (9) and (10). It remains to prove that we have

$$
\operatorname{val}(D)=\left(\frac{M\left(x_{0}\right)-m I\left(x_{0}\right)}{I\left(x_{0}\right) M\left(x_{1}\right)-I\left(x_{1}\right) M\left(x_{0}\right)}\right) \int_{0}^{x_{1}} \psi(u) f(u) d u
$$

and that the measure $\mu$ defined by

$$
d \mu \triangleq\left[\left(\frac{M\left(x_{1}\right)-m I\left(x_{1}\right)}{I\left(x_{0}\right) M\left(x_{1}\right)-I\left(x_{1}\right) M\left(x_{0}\right)}\right) \mathbf{1}_{\left(0, x_{0}\right)}+\left(\frac{M\left(x_{0}\right)-m I\left(x_{0}\right)}{M\left(x_{0}\right) I\left(x_{1}\right)-I\left(x_{0}\right) M\left(x_{1}\right)}\right) \mathbf{1}_{\left(0, x_{1}\right)}\right] d x
$$


is in $\operatorname{Sol}(P)$, for any couple $\left(x_{0}, x_{1}\right) \in \mathbb{R}^{2}$ which satisfies the conditions (8), (9) and (10). Let $\left(x_{0}, x_{1}\right)$ be such a couple. Then, on the one hand, by (8) and (9) and from Lemma B.8, there exists $(\alpha, \beta) \in(0, \infty)^{2}$ such that

$$
\begin{aligned}
& \alpha \int_{0}^{x_{0}} f(u) d u+\beta \int_{0}^{x_{1}} f(u) d u=1 \\
& \alpha \int_{0}^{x_{0}} u f(u) d u+\beta \int_{0}^{x_{1}} u f(u) d u=m \\
& \alpha \int_{0}^{x_{0}} u^{2} f(u) d u+\beta \int_{0}^{x_{1}} u^{2} f(u) d u=\delta
\end{aligned}
$$

and

$$
\beta=\frac{M\left(x_{0}\right)-m I\left(x_{0}\right)}{I\left(x_{0}\right) M\left(x_{1}\right)-I\left(x_{1}\right) M\left(x_{0}\right)} .
$$

It follows that, for all $v \in \mathcal{A}$,

$$
\begin{aligned}
v_{0}+v_{1} m+v_{2} \delta & =\alpha \int_{0}^{x_{0}}\left(v_{0}+v_{1} u+v_{2} u^{2}\right) f(u) d u+\beta \int_{0}^{x_{1}}\left(v_{0}+v_{1} u+v_{2} u^{2}\right) f(u) d u \\
& \geq \alpha \int_{0}^{x_{0}} \psi(u) f(u) d u+\beta \int_{0}^{x_{1}} \psi(u) f(u) d u
\end{aligned}
$$

On the other hand, by (9) and (10), and from Lemma B.9, there exists $\lambda \in \mathcal{A}$ such that $\operatorname{bind}(\lambda)=\left\{x_{0}, x_{1}\right\}$. The equality therefore holds for $\lambda$, i.e.

$$
\begin{aligned}
\lambda_{0}+\lambda_{1} m+\lambda_{2} \delta & =\alpha \int_{0}^{x_{0}}\left[\lambda_{0}+\lambda_{1} u+\lambda_{2} u^{2}\right] f(u) d u+\beta \int_{0}^{x_{1}}\left[\lambda_{0}+\lambda_{1} u+\lambda_{2} u^{2}\right] f(u) d u \\
& =\alpha \int_{0}^{x_{0}} \psi(u) f(u) d u+\beta \int_{0}^{x_{1}} \psi(u) f(u) d u
\end{aligned}
$$

It ensues then from (B.30), from the fact that $x_{0}<K$ and from (B.29), that

$$
\begin{aligned}
\operatorname{val}(D) & =\alpha \int_{0}^{x_{0}} \psi(u) f(u) d u+\beta \int_{0}^{x_{1}} \psi(u) f(u) d u \\
& =\beta \int_{0}^{x_{1}} \psi(u) f(u) d u \\
& =\frac{M\left(x_{0}\right)-m I\left(x_{0}\right)}{I\left(x_{0}\right) M\left(x_{1}\right)-I\left(x_{1}\right) M\left(x_{0}\right)} \int_{0}^{x_{1}} \psi(u) f(u) d u
\end{aligned}
$$


Finally, it is easy to check that the measure $\mu$ defined by

$$
d \mu \triangleq\left[\left(\frac{M\left(x_{1}\right)-m I\left(x_{1}\right)}{I\left(x_{0}\right) M\left(x_{1}\right)-I\left(x_{1}\right) M\left(x_{0}\right)}\right) \mathbf{1}_{\left(0, x_{0}\right)}+\left(\frac{M\left(x_{0}\right)-m I\left(x_{0}\right)}{M\left(x_{0}\right) I\left(x_{1}\right)-I\left(x_{0}\right) M\left(x_{1}\right)}\right) \mathbf{1}_{\left(0, x_{1}\right)}\right] d x
$$

is in $\mathcal{C}_{m, \delta}$ and that we have

$$
\int_{0}^{\infty} \psi(x) f(x) d \mu(x)=\frac{M\left(x_{0}\right)-m I\left(x_{0}\right)}{I\left(x_{0}\right) M\left(x_{1}\right)-I\left(x_{1}\right) M\left(x_{0}\right)} \int_{0}^{x_{1}} \psi(u) f(u) d u
$$

so that $\mu \in \operatorname{Sol}(P)$. This ends the proof of Theorem 3.2 (ii) and completes the proof of Theorem 3.2.

\section{B.4 Proof of Proposition 3.1}

As the proof is very similar to the one of Theorem 3.1, we only give a sketch of it. First it is shown that

$$
\sup _{\mu \in \mathcal{C}_{m}} \int_{0}^{\infty} \psi(x) f(x) d \mu(x) \leq \inf _{\lambda \in \mathcal{A}_{2}} \lambda_{0}+\lambda_{1} m
$$

where $\mathcal{A}_{2}$ is the set of $\lambda \in \mathbb{R}^{2}$ satisfying $\int_{0}^{x}\left[\lambda_{0}+\lambda_{1} u-\psi(u)\right] f(u) d u \geq 0$ for all $x \in \mathbb{R}^{+}$. It is easy to see that, if $\lambda \in \mathcal{A}_{2}$ then $\lambda_{0} \geq 0$. Then recalling that $m \leq p_{1}$, one shows that, for all $\lambda \in \mathcal{A}_{2}$, we have

$$
\lambda_{0}+\lambda_{1} m \geq\left(m / p_{1}\right)\left(\lambda_{0}+\lambda_{1} p_{1}\right) \geq\left(m / p_{1}\right)\left(\int_{0}^{\infty} \psi(x) f(x) d x\right) .
$$

The proof is completed in the same way as the proof of Theorem 3.1 by considering $\lambda \triangleq\left(0, \frac{\int_{0}^{\infty} \psi(x) f(x) d x}{p_{1}}\right)$.

\section{References}

Bertsimas D. and I. Popescu (2002) On the relation between option and stock prices: an optimization approach, Operations Research, 50, 358-374.

Basso A. and P. Pianca (2001) Option pricing bounds with standard risk aversion preferences, European Journal of Operational Research, 134, 249-260. 
Bizid A., E. Jouini and P.-F. Koehl (1999) Pricing of nonredundant asset in a complete market, Review of Derivatives Research, 2, 287-314.

Constantinides G.M. and S. Perrakis (2002) Stochastic dominance bounds on derivatives prices in a multiperiod economy with proportional transaction costs, Journal of Economic Dynamics and Control, 26, 1323-1352.

Gotoh J. and H. Konno (2002) Bounding option prices by semidefinite programming: a cutting plane algorithm, Management Science, 48, 66-678.

Grundy B. (1991) Option prices and the underlying asset's return distribution, Journal of Finance, 46, 1045-1069.

Jouini E. (2003) Convergence of the equilibrium prices in a family of financial models, Finance and Stochastics, 7, 491-507.

Jouini E. and C. Napp (1998) Continuous time equilibrium pricing of nonredundant assets, Working Paper 9830, CREST.

Jouini E. and C. Napp (2003) A class of models satisfying a dynamical version of the CAPM, Economics Letters, 79, 299-304.

Karatzas I. (1989) Optimization problems in theory of continuous trading, SIAM J. Control. Optimization, 27, 1221-1259.

Levy H. (1985) Upper and lower bounds of put and call option value: stochastic dominance approach, Journal of Finance, 40, 1197-1217.

lo Lo A. (1987) Semi-parametric upper bounds for option prices and expected payoffs, Journal of Financial Economics, 19, 373-387.

Perrakis S. and P.J. Ryan (1984) Option pricing bounds in discrete time, Journal of Finance, 39, 519-525.

Popescu I. (2002) A semidefinite programming approach to optimal moment bounds for distributions with convex properties, INSEAD working paper, 2002/54/TM.

Ritchken P.H. (1985) On option pricing bounds, Journal of Finance, 40, 1219-1233. 
Ritchken P.H. and S. Kuo (1989) Stochastic Dominance and Decreasing Absolute Risk Averse Option Pricing Bounds, Management Science, 35, 51-59.

Shapiro A. (2001) On duality theory of conic linear problems, Miguel A. Goberna and Marco A. Lopez, Eds., Semi-Infinite Programming: Recent Advances, Kluwer Academic Publishers, 135-165. 
Table 1. Black-Scholes price, equilibrium bound with 2 moment constraints, equilibrium bound with 1 moment constraint (Perrakis and Ryan), bound with 4 moment constraints, bound with 2 moment constraints (Lo), for different strike prices and volatilities.

\begin{tabular}{|c|c|c|c|c|c|c|}
\hline$\sigma$ & $K$ & $\mathrm{BS}$ & $\operatorname{val}(P)(e)$ & $B_{P \& R}(e)$ & $B_{4}(e)$ & $B_{L o}(e)$ \\
\hline \hline \multirow{6}{*}{$60 \%$} & 300 & 125.513 & $129.649(3 \%)$ & $135.177(8 \%)$ & $134.666(7 \%)$ & $154.877(23 \%)$ \\
& 350 & 94.110 & $97.466(4 \%)$ & $103.291(10 \%)$ & $104.057(11 \%)$ & $119.874(27 \%)$ \\
& 400 & 69.324 & $71.312(3 \%)$ & $77.542(12 \%)$ & $81.384(17 \%)$ & $90.668(31 \%)$ \\
& 450 & 50.411 & $51.290(2 \%)$ & $57.444(14 \%)$ & $64.727(28 \%)$ & $68.241(35 \%)$ \\
& 500 & 36.330 & $37.024(2 \%)$ & $42.152(16 \%)$ & $51.740(42 \%)$ & $52.191(44 \%)$ \\
\hline \multirow{6}{*}{$40 \%$} & 300 & 113.803 & $117.350(3 \%)$ & $125.201(10 \%)$ & $118.335(4 \%)$ & $131.506(16 \%)$ \\
& 350 & 76.575 & $79.417(4 \%)$ & $87.412(14 \%)$ & $82.468(8 \%)$ & $92.648(21 \%)$ \\
& 400 & 48.323 & $49.224(2 \%)$ & $57.449(19 \%)$ & $57.222(18 \%)$ & $61.100(26 \%)$ \\
& 450 & 28.862 & $33.476(14 \%)$ & $35.792(24 \%)$ & $39.533(37 \%)$ & $39.636(37 \%)$ \\
& 500 & 16.487 & $21.223(22 \%)$ & $21.328(29 \%)$ & $25.224(53 \%)$ & $27.038(64 \%)$ \\
\hline \multirow{6}{*}{$20 \%$} & 300 & 108.353 & $110.696(2 \%)$ & $121.41(12 \%)$ & $109.349(1 \%)$ & $114.692(6 \%)$ \\
& 350 & 62.446 & $64.935(4 \%)$ & $76.265(22 \%)$ & $64.310(3 \%)$ & $70.180(12 \%)$ \\
& 400 & 27.274 & $28.705(5 \%)$ & $38.137(40 \%)$ & $32.001(17 \%)$ & $33.304(22 \%)$ \\
& 450 & 8.633 & $9.609(11 \%)$ & $14.315(66 \%)$ & $12.874(49 \%)$ & $14.324(66 \%)$ \\
& 500 & 2.014 & $2.239(11 \%)$ & $4.022(100 \%)$ & $3.424(70 \%)$ & $7.908(293 \%)$ \\
\hline
\end{tabular}

